\title{
A PDF-Based Formulation of Microphysical Variability in Cumulus Congestus Clouds*
}

\author{
YEFIM L. KOGAN \\ Cooperative Institute for Mesoscale Meteorological Studies, University of Oklahoma, Norman, Oklahoma \\ DAVID B. MECHEM \\ Atmospheric Science Program, Department of Geography, University of Kansas, Lawrence, Kansas
}

(Manuscript received 12 May 2015, in final form 13 August 2015)

\begin{abstract}
Calculating unbiased microphysical process rates over mesoscale model grid volumes necessitates knowledge of the subgrid-scale (SGS) distribution of variables, typically represented as probability distribution functions (PDFs) of the prognostic variables. In the 2014 Journal of the Atmospheric Sciences paper by Kogan and Mechem, they employed large-eddy simulation of Rain in Cumulus over the Ocean (RICO) trade cumulus to develop PDFs and joint PDFs of cloud water, rainwater, and droplet concentration. In this paper, the approach of Kogan and Mechem is extended to deeper, precipitating cumulus congestus clouds as represented by a simulation based on conditions from the TOGA COARE field campaign. The fidelity of various PDF approximations was assessed by evaluating errors in estimating autoconversion and accretion rates. The dependence of the PDF shape on grid-mean variables is much stronger in congestus clouds than in shallow cumulus. The PDFs obtained from the TOGA COARE simulations for the calculation of accretion rates may be applied to both shallow and congestus cumulus clouds. However, applying the TOGA COARE PDFs to calculate autoconversion rates introduces unacceptably large errors in shallow cumulus clouds, thus precluding the use of a "universal" PDF formulation for both cloud types.
\end{abstract}

\section{Introduction}

It is well known that neglecting subgrid-scale (SGS) variability can lead to substantial bias in calculations of microphysical process rates (Pincus and Klein 2000; Larson et al. 2001; Wood et al. 2002; Larson et al. 2012; Kogan and Mechem 2014). The rate bias is particularly large for autoconversion, which is typically formulated as a convex function of cloud water mixing ratio $q_{c}$ and droplet concentration $N_{c}$. For a convex function, Jensen's inequality tells us that $\overline{f\left(q_{c}, N_{c}\right)}>f\left(\overline{q_{c}}, \overline{N_{c}}\right)$, where the overbar represents grid-mean quantities. Autoconversion aims to represent precipitation initiation

\footnotetext{
* Supplemental information related to this paper is available at the Journals Online website: http://dx.doi.org/10.1175/JAS-D-150129.s1.

Corresponding author address: Yefim Kogan, Cooperative Institute for Mesoscale Meteorological Studies, University of Oklahoma, 120 David L. Boren Blvd., Suite 2100, Norman, OK 73072-7304. E-mail: ykogan@ou.edu
}

through the formation of small precipitation-sized droplets from smaller cloud droplets. The formation of embryonic precipitation drops is energetically unfavorable and represents a bottleneck in the classical warmrain process (Beard and Ochs 1993). For this reason, autoconversion can exert a substantial influence on the precipitation process, and any bias in autoconversion rate can lead to biases in precipitation rate, liquid water path, entrainment rate, and thermodynamic stratification of the boundary layer (Larson et al. 2012). Autoconversion rate bias arising from the neglect SGS variability is negative, suggesting a suppression of precipitation and reduction of cloud-system behavior associated with precipitation. These microphysical responses of suppressed precipitation suggest a reduction of mesoscale variability observationally associated with precipitation (Comstock et al. 2005), as well as reduced aerosol feedbacks (Mechem et al. 2006; Wood 2006).

The solution to the SGS variability bias problem lies in representing the variability using probability distribution functions (PDFs) for the different variables. The microphysical process rates are integrated over these 
PDFs-an operation that is equivalent to taking the sum of the local microphysical process rates everywhere over the domain. The greatest difficulty in this approach lies in the choice of PDF shape and the closure assumptions required to link the PDF parameters to the grid-mean prognostic variables. Kogan and Mechem (2014, hereafter KM2014) summarize several of these recent PDF approaches - the most sophisticated of which employs joint analytic PDFs of liquid water potential temperature, total water, and vertical velocity [Cloud Layers Unified by Binomials (CLUBB); Golaz et al. 2002; Larson et al. 2002; Larson and Golaz 2005]. The CLUBB approach uses theoretical considerations and a number of a priori assumptions about the shape of the distributions, established from LES output and aircraft observations. KM2014 propose a method that establishes PDFs and closure assumptions formulated from a series of large-eddy simulations of precipitating shallow cumulus based on the Rain in Cumulus over the Ocean (RICO; Rauber et al. 2007) field campaign. The KM2014 methodology includes two distinct elements for deriving the PDF parameterization. The first is the use of a layered approach to define the vertical variation of the PDF, which increases the statistical robustness of the PDFs. The second element is the formulation of the PDFs in nondimensional space by normalizing the microphysical variables by their layer-mean values.

Along with deep convection, trade cumulus, such as those sampled in the RICO campaign, constitute one of the two distinct modes of tropical cloudiness. The existence of a third mode-cumulus congestus-was suggested in early studies by Malkus (1962) and Malkus and Riehl (1964). Johnson et al. (1999) found robust signs of cumulus congestus during the Tropical Ocean and Global Atmosphere Coupled Ocean-Atmosphere Response Experiment (TOGA COARE; Godfrey et al. 1998). Other studies have identified congestus from surface-based millimeter-wavelength radar (Hollars et al. 2004; Jensen and Del Genio 2006; Stephens and Wood 2007) and spacebased radar (Casey et al. 2007, 2012; Luo et al. 2009).

Congestus may constitute a substantial source of tropical precipitation, although the exact contribution of congestus to the total tropical precipitation is not well constrained. Congestus contributions from previous studies include values of 20\% (Liu and Zipser 2009), 27.3\% (Stephens and Wood 2007), 28\% (Johnson et al. 1999), and 30\% (Cheng and Houze 1979)—estimates that come from a number of different observational platforms. Mechem and Oberthaler (2013) summarize some of the methodological differences behind these different estimates and find a congestus contribution of $34 \%$ based on simulations from a 10 -day period during TOGA COARE.
The congestus contribution to surface precipitation constitutes a "bottom heavy" latent heating influence on the large-scale tropical atmosphere ( $\mathrm{Li}$ et al. 2009), which may be consistent with better representation of the Madden-Julian oscillation (MJO; Madden and Julian 1971, 1972). Furthermore, congestus clouds act to moisten and destabilize the tropical atmosphere (Waite and Khouider 2010), although Hohenegger and Stevens (2013) argue that this destabilization mechanism is not active at hourly time scales. Mechem and Oberthaler (2013) found that, in many ways, congestus act like overgrown trade cumulus, suggesting that similarity scaling approaches (e.g., Khairoutdinov and Randall 2002) may be able to represent both cloud types.

As an important aspect of the MJO transition and because of their large contribution to surface precipitation, congestus need to be accurately represented in numerical models. Accounting for their SGS variability therefore is no less important than for shallow trade cumulus. In this paper, we address the question of PDF variability of congestus clouds by extending the PDF methodology of KM2014 to cumulus congestus clouds. For this purpose, we use high-resolution, bulkmicrophysics simulations of TOGA COARE cumulus congestus based on a subset of the period investigated by Mechem and Oberthaler (2013).

Our approach includes formulating joint PDFs of the microphysical variables, testing a hierarchy of parameterizations of various degrees of complexity, and evaluating the generality of the PDF parameterization for cases of both congestus and trade cumulus.

\section{Model and dataset}

We employ a version of the Cooperative Institute for Mesoscale Meteorological Studies (CIMMS) LES (Kogan et al. 1995; Khairoutdinov and Kogan 1999) called System for Atmospheric Modeling-Bulk Microphysics (SAMBM) (Kogan et al. 2012) to develop a suite of PDFs of cumulus congestus. The System for Atmospheric Modeling (SAM; Khairoutdinov and Randall 2003) forms the dynamical core of SAMBM. SAM is based on anelastic dynamics and monotonic, positive-definite advection for scalars (Smolarkiewicz and Grabowski 1990). The prognostic bulk microphysical variables include $\mathrm{CCN}$ count $N_{\text {ccn }}$, cloud water mixing ratio $q_{c}$, cloud-drop concentration $N_{c}$, integral radius of cloud droplets $R_{c}$, drizzle water mixing ratio $q_{r}$, and drizzle drop concentration $N_{r}$. The bulk microphysical parameterization for shallow cumulus from Kogan (2013) is employed. This microphysical parameterization was developed based on large-eddy simulation drop size distribution output for a case derived from 


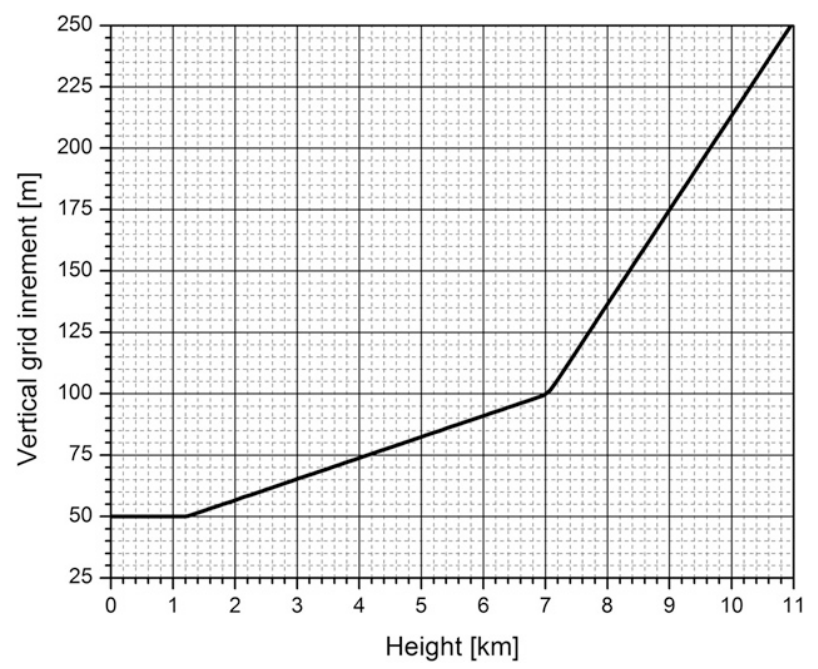

FIG. 1. Vertical grid increments used in simulation.

the RICO (Rauber et al. 2007; vanZanten et al. 2011) trade cumulus field campaign.

SAMBM simulations have been evaluated against simulation output from the CIMMS LES model using size-resolving (bin) microphysics (SAMEX; Kogan et al. 2012). The explicit microphysics formulation of SAMEX uses 34 cloud drop bins ranging in size from $1 \mu \mathrm{m}$ up to $2 \mathrm{~mm}$ and $19 \mathrm{CCN}$ bins that range from aerosol dry radius size of $0.076-5.5 \mu \mathrm{m}$.

The simulations used to develop and evaluate PDFs were drawn from the 10-day analysis period in Mechem and Oberthaler (2013). The LES was initialized with profiles of liquid water potential temperature, total water, and wind components corresponding to conditions from 9 December 1992 [day-of-year (DOY) 344] of the TOGA COARE field campaign. The profiles were derived from the TOGA COARE observations using the variational analysis method of Zhang and Lin (1997). The variational analysis product also provided the observationally constrained vertical motion field and the large-scale advection of temperature and moisture, which are imposed as forcing for the simulation.

DOY 344 corresponds to the very end of the suppressed period of the MJO, just prior to the onset of the active phase. The horizontal grid spacing for the simulation was $100 \mathrm{~m}$ - the same as the grid spacing used in the RICO trade cumulus intercomparison (vanZanten et al. 2011). All simulations employed a stretched vertical grid similar to that of Mechem and Oberthaler (2013). The vertical grid increment was $50 \mathrm{~m}$ in the lower $1.2 \mathrm{~km}$, then increased linearly to $100 \mathrm{~m}$ up to a height of $7 \mathrm{~km}$ (the top of the majority of the tallest clouds), and further increased to $250 \mathrm{~m}$ at the top of the domain (Fig. 1). Both Mechem and Oberthaler (2013) and

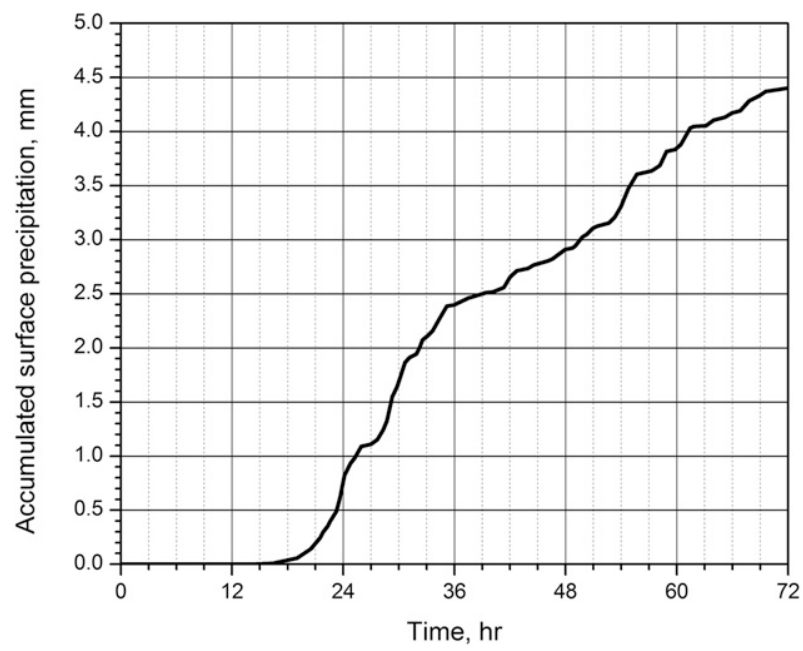

FIG. 2. Evolution of accumulated precipitation at the surface.

Khairoutdinov et al. (2009) found that high vertical resolution was required to adequately represent the congestus mode. A total of $384 \times 384 \times 130$ grid points was used for the domain size spanning $38.4 \times 38.4 \times$ $11 \mathrm{~km}^{3}$. The $38.4 \times 38.4 \mathrm{~km}^{2}$ domain size is roughly equivalent to a single coarse-mesh grid volume of a current mesoscale model. The $384 \times 384$ finescale horizontal grid points in our domain allow us to study in detail the subgrid variability across this domain. The horizontal-layer-mean variables in our domain, therefore, correspond to prognostic grid-scale (resolvable) variables of a mesoscale model. Additional simulations are required to evaluate the dependence of variability on mesoscale model grid resolution, but this is beyond the scope of our study and will be the subject of a separate investigation.

The 72-h simulation used a 2-s dynamical time step. The time series of the accumulated precipitation at the surface during the simulation is shown in Fig. 2. After an initial 16-h spinup period, the precipitation increased rapidly during the $17-36-\mathrm{h}$ period and then remained at a slower domain-average rate of $1.3 \mathrm{~mm} \mathrm{day}^{-1}$ for the rest of simulation. Simulation output from the 36-72-h period was included in the dataset, which contained over $2 \times 10^{6}$ cloudy data points for analysis. Cumulative distributions from ASTEX, RICO, and TOGA simulations (obtained by counting all cloudy pixels in each of the three simulations) differ substantially, as would be expected (Fig. 3). Clouds in the TOGA simulation are taller, contain more liquid water, and produce more precipitation.

As in KM2014, in order to attribute precipitation to clouds of different thickness, we divide the dataset into four groups (Fig. 4). These four groups are based on 

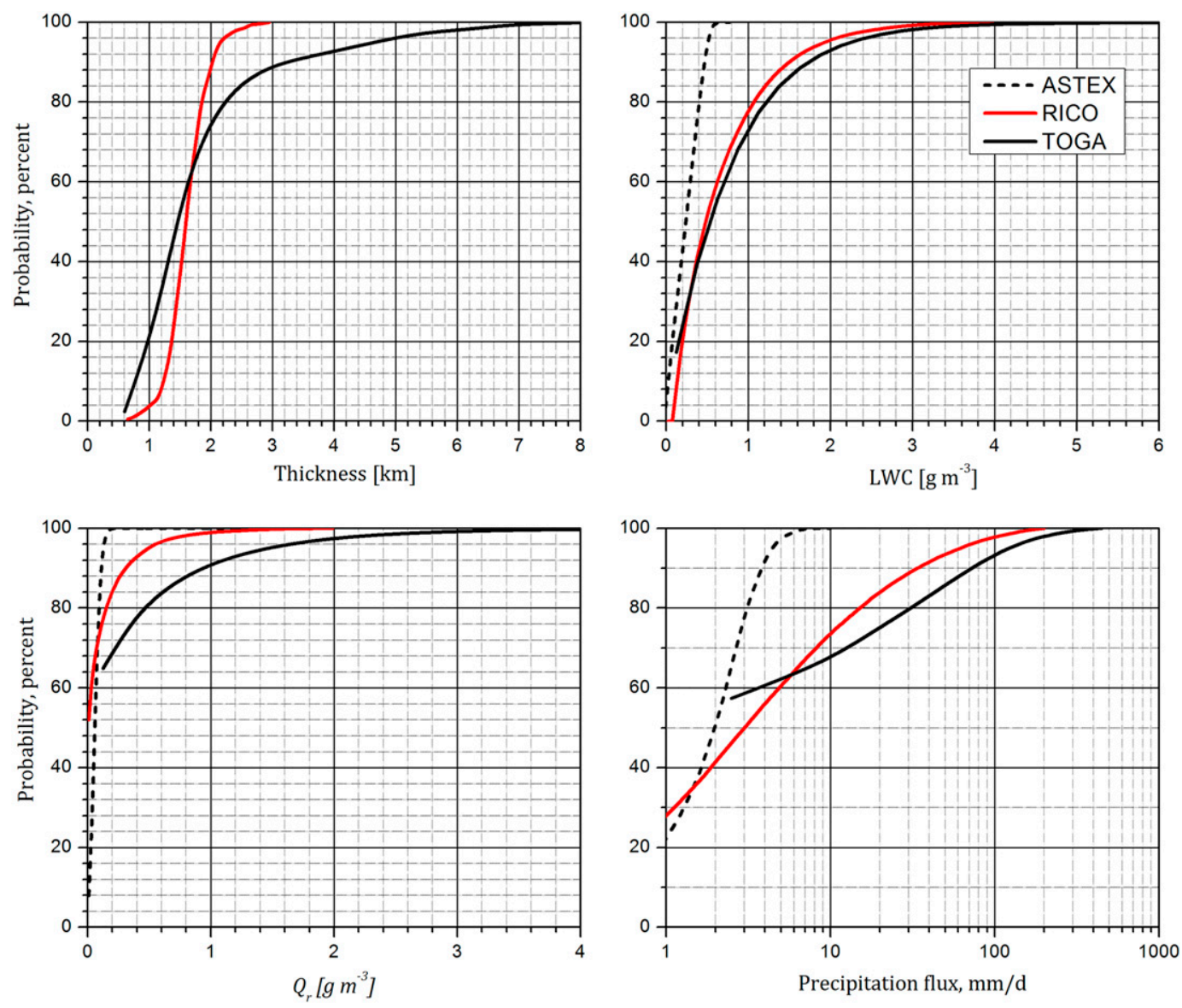

FIG. 3. Comparison of parameters of cloud systems in ASTEX, RICO, and TOGA simulations: cumulative distributions of (top left) cloud thickness, (top right) liquid water content, (bottom left) rainwater, and (bottom right) precipitation flux. The ASTEX cloud thickness is a nearly constant value of $\sim 0.3 \mathrm{~km}$ and is not plotted.

quartiles of the cumulative cloud-top distribution, with each group containing approximately equal numbers of clouds (the use of stretched vertical grid with grid increments between 50 and $250 \mathrm{~m}$ precludes an exactly even distribution across the four categories). The top quartile (group 4) includes clouds with tops higher than $2.7 \mathrm{~km}$. The $10 \%$ of the cloud population has tops extending above $4 \mathrm{~km}$. These TOGA congestus clouds are far deeper than clouds in the RICO simulation (vanZanten et al. 2011; KM2014), which were lower than $3.1 \mathrm{~km}$. In the text below, we refer to the four groups as G1-G4.

Figure 5a shows that in the TOGA case clouds in the G4 category constitute about $65 \%$ of the total horizontal cross section, $81 \%$ of the total cloudy volume, and $85 \%$ of the total precipitation. The contrast between G4 clouds and the shallower G1, G2, and G3 categories in the TOGA simulation is more pronounced than in the RICO simulation. In the RICO case, the percentages of the G4 clouds in the area, volume, and precipitation are about $46 \%, 56 \%$, and $71 \%$ (Fig. $5 b$ ). The overwhelming precipitation contribution from the G4 clouds in the TOGA simulation is a result from a larger precipitation rate associated with the deeper TOGA clouds and the wider horizontal cross section. The total precipitation flux produced by clouds in the group G1 is a tiny $0.3 \%$ of the amount of G3 and G4 clouds, and clouds in the G1 and $\mathrm{G} 2$ categories contribute only $2.5 \%$, suggesting that these shallow clouds contribute very little to the precipitation process. For this reason the development of our PDF parameterization will be based on the analysis of clouds from groups G3 and G4. Even though the precipitation contribution from the smaller G1 and G2 clouds is insignificant; nevertheless, their contribution to cloud cover is substantial (about 16\%; see area parameter in Fig. 5a), and these smaller clouds cannot be neglected in studies addressing cloud albedo. Furthermore, these shallower clouds likely also serve to moisten, cool, and deepen the boundary layer, in a manner similar to trade cumulus (Mechem and 


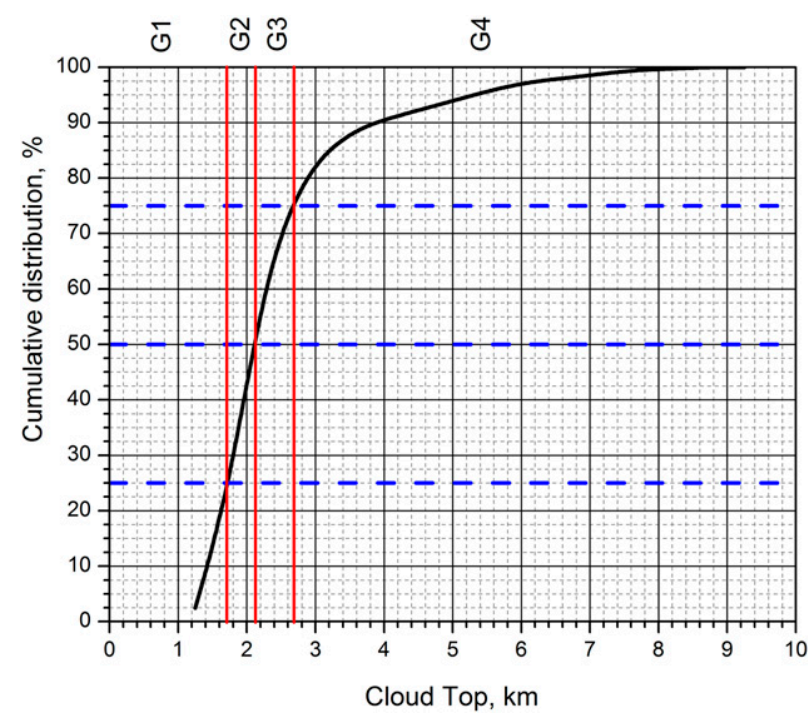

FIG. 4. Cumulative distribution of cloud-top heights. Dashed lines mark $25 \%, 50 \%$, and $75 \%$ quartiles.

Oberthaler 2013). These latter effects, however, are beyond the scope of our current investigation.

Nearly all cloud tops $(>99 \%)$ lie lower than $8 \mathrm{~km}$ (Fig. 4). We use the clouds from the G3 and G4 groups to formulate height-dependent PDFs, which are discretized into 10 layers spanning from cloud base $(\sim 0.6 \mathrm{~km})$ to cloud top $(\sim 7 \mathrm{~km})$. Only a few cloud tops exceed $7 \mathrm{~km}$, and the total number of cloudy points at these high levels does not exceed the 100-gridpoint threshold requirement to calculate the PDF. Because of the stretched vertical grid, layer thickness increases from $500 \mathrm{~m}$ near cloud base (levels 1-3), to about $600 \mathrm{~m}$ in the middle of the cloud layer, and up to $870 \mathrm{~m}$ in the upper part of the cloud. These thick layers combine multiple vertical model levels, which substantially increases the number of data points for each PDF calculation and thus our statistical confidence in the calculations. For G4 clouds, layers 1 and 2 are near cloud base, layers 3-7 cover the middle of the clouds, and layers 8-10 represent regions near cloud top.

As the TOGA cloud system evolves, the horizontallayer-mean cloud properties vary with time. Ideally, we would like to analyze each of the 36 hourly datasets separately to obtain PDFs that are time dependent. Importance of time evolution of PDFs (in other words, their dependence on horizontal grid-mean variables) was studied in KM2014 using the hourly datasets from RICO simulation. The results (Figs. 13a and 14a in KM2014) demonstrated that using PDFs that are fixed in time (i.e., neglecting dependence of PDFs on horizontal grid-mean variables) only insignificantly increases errors of conversion rates. For example, in
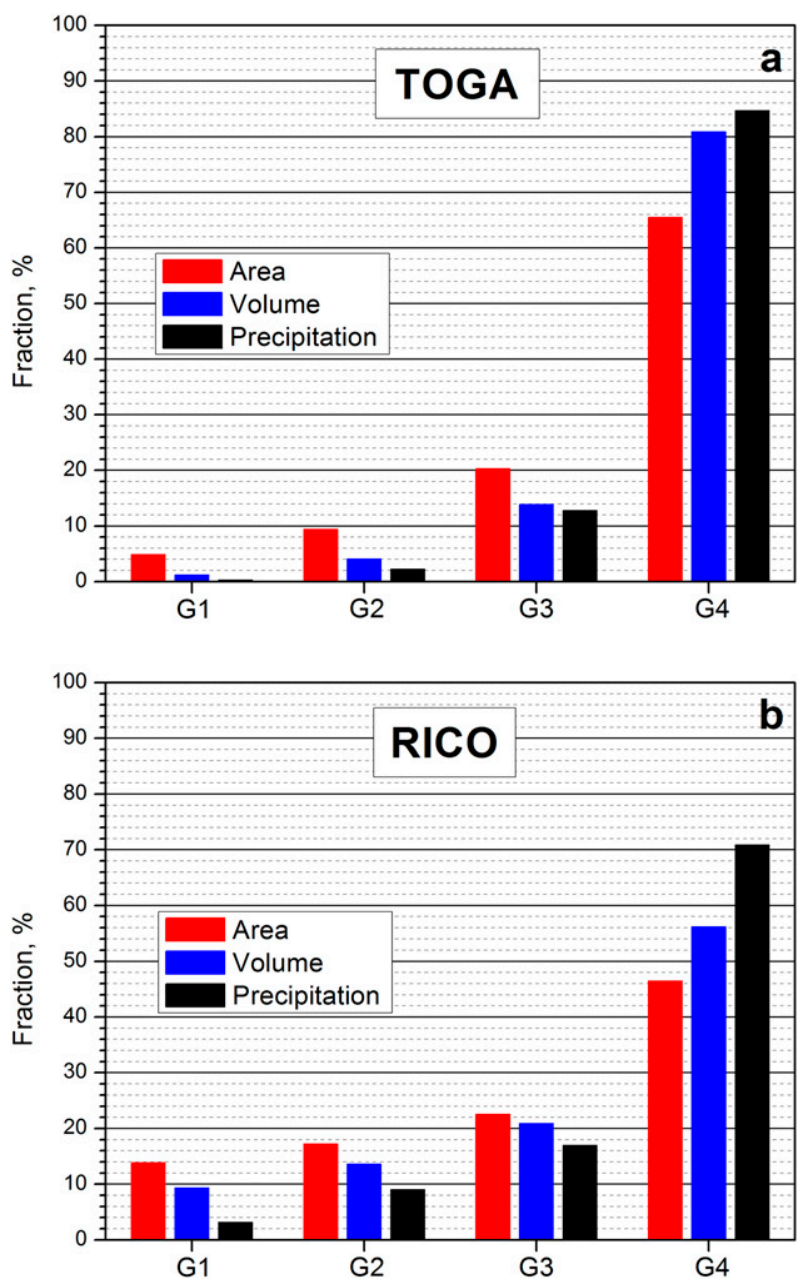

FIG. 5. Statistics of cloud macro parameters by group in (a) TOGA and (b) RICO simulations. Parameters are shown as percentages of their totals for all groups. "Area" refers to the sum of horizontal cross sections of all clouds in a group, "volume" refers to the sum of all cloudy points in a group, and "precipitation" refers to the sum of precipitation flux at cloud base in a group.

estimating the autoconversion rate using fixed time PDFs, the mean error increased from $7.5 \%$ to $10.1 \%$. For accretion rates, the mean error increased from $10.5 \%$ to $13.9 \%$. KM2014 demonstrated that accounting for the vertical variation of the PDFs was substantially more important than representing their temporal evolution. We will show, however, that the time dependence of the PDFs is much stronger in the TOGA case-an outcome that will be discussed in section 5 where we will evaluate the accuracy of various PDF approximations.

\section{One-dimensional PDFs}

Although we are predominantly interested in joint PDFs, we first calculate 1D PDFs of individual model 

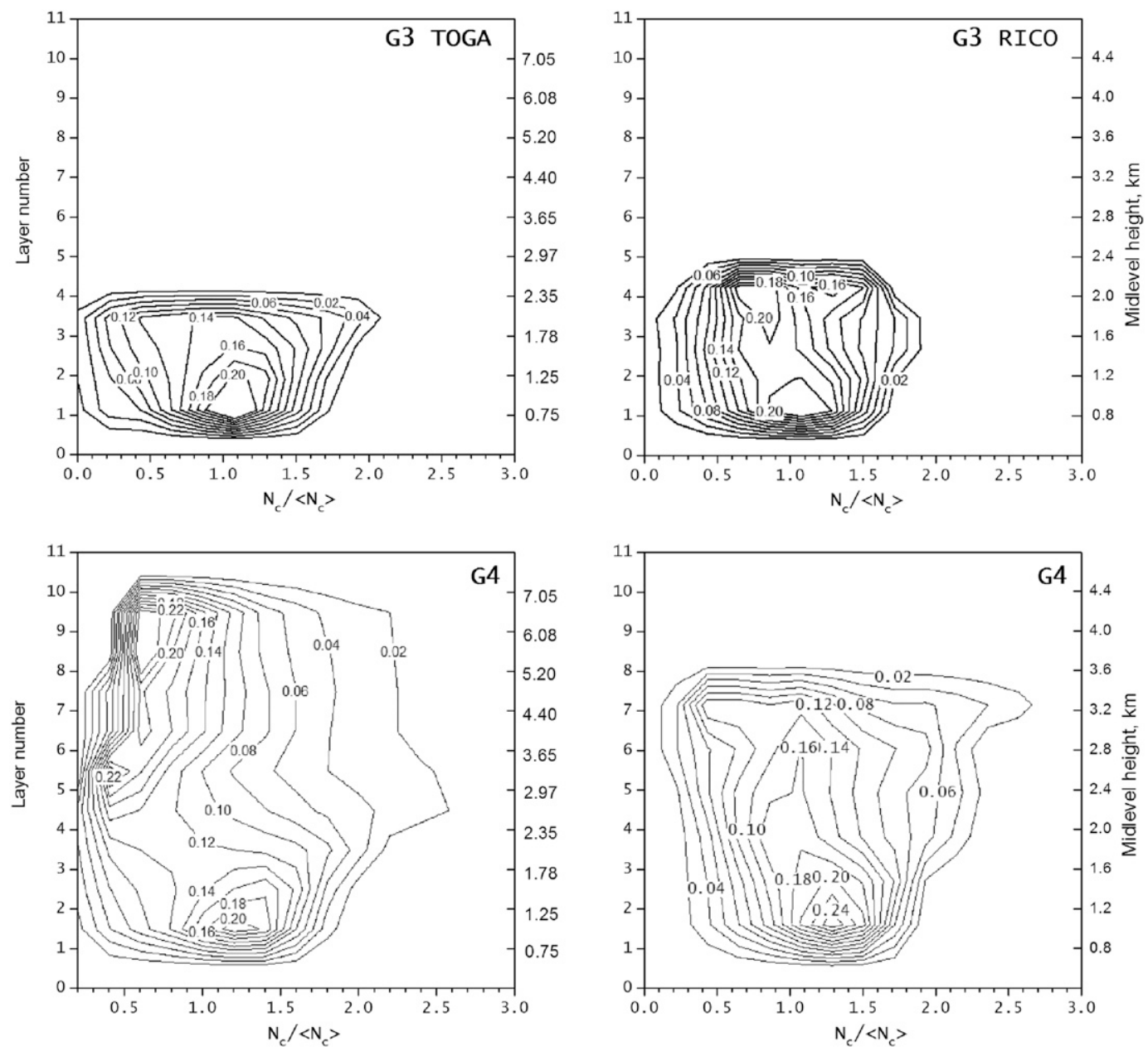

FIG. 6. Isolines of PDFs of $N_{c}$ as a function of vertical layer for cloud groups G3 and G4 in the (left) TOGA and (right) RICO simulations. The $x$ axis represents the PDF distribution variable normalized by its layer-mean value. The $x$ axis represents $N_{c} /\left\langle N_{c}\right\rangle$. The left $y$ axis is the vertical layer number; the corresponding height of the level's midpoint is shown on the right $y$ axis.

variables (cloud drop number concentration $N_{c}$, cloud water mixing ratio $q_{c}$, and rainwater mixing ratio $q_{r}$ ). In addition to helping better understand the variability of these cloud properties, 1D PDFs (particularly of the cloud water mixing ratio) are particularly useful for calculating unbiased autoconversion rates in singlemoment microphysical parameterizations. We formulate the PDFs in each of the layers by first normalizing each variable and then binning into 15 regularly spaced bins ranging from 0 to 3 (nondimensional values). Over each layer, we required a threshold number of 100 points in order to be included in the PDF calculation-a requirement often not met in the topmost layers.

Figure 6 demonstrates the shape of a 1D PDF of $N_{c}$ and its variation over the 10 vertical layers by displaying PDF isolines as a function of $N_{c}$ and the vertical layer $L$. Qualitatively, the PDF for the TOGA group G4 is less symmetric than the PDFs derived from the RICO trade cumulus simulations (KM2014), with the mode of the PDF at each level decreasing with height in both TOGA and RICO cases. The somewhat negative skewness of the PDFs at the lower layers (more pronounced in the TOGA case) reflects the fact that at cloud base majority of $N_{c}$ concentrations is larger than layer-average $N_{c}$, while the opposite is true for cloud top. Physically, this pattern reflects the activation of new drops near cloud base (leading to the increase in $N_{c}$ ) and decrease in $N_{c}$ owing to coagulation at cloud top. It is interesting to note the pronounced maximum near the middle layers for the TOGA G4 clouds, while no such maximum exists for G4 RICO clouds. This feature is probably a sampling artifact of the G4 TOGA clouds and is not statistically meaningful. In particular, from Fig. 4 it is clear that G4 group contains clouds with tops between 

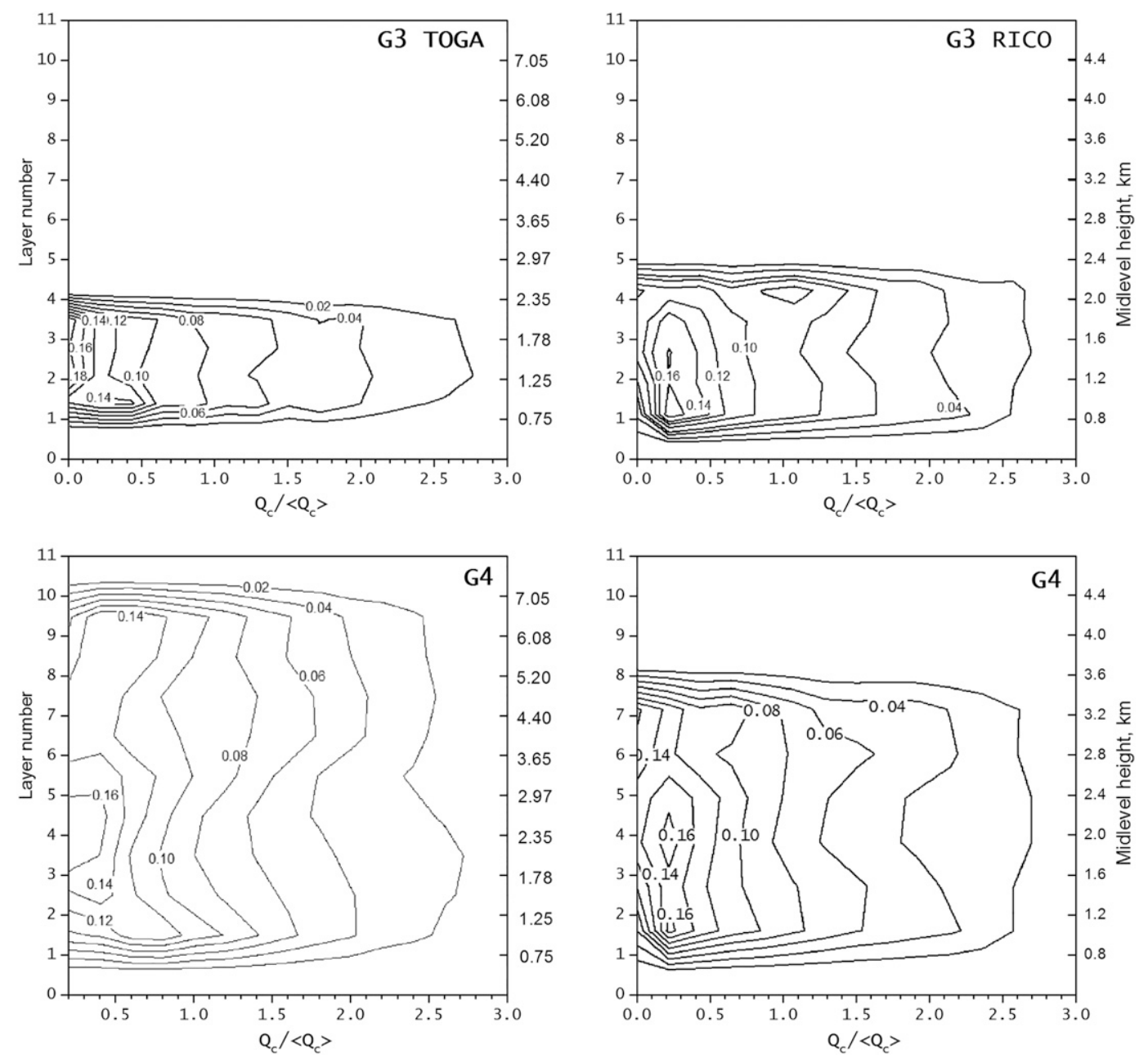

FIG. 7. As in Fig. 6, but for cloud water mixing ratio.

2.7 and $8 \mathrm{~km}$. As a result, the sixth cloud layer (centered around $3.6 \mathrm{~km}$ ) might sample, for example, both the top of a 4-km-high cloud and the middle of a 7-km-high cloud, both from the G4 group. Groups G1-G3 include a more narrow range of cloud-top heights; therefore, their PDFs have a simpler, more obvious physical interpretation.

Distributions of $q_{c}$ exhibit a significant positive skewness (Fig. 7) at all levels. The vertical variation of the PDFs is somewhat less than for $N_{c}$. Notably, PDFs of $q_{c}$ are broader than PDFs of $N_{c}$. The physical explanation may be due to the fact that $q_{c}$ is proportional both to $N_{c}$ and the cube of drop radius, and therefore condensational growth in rising updrafts will rapidly broaden the $q_{c}$ distribution. The growth of $q_{c}$ by condensation is limited, on the other hand, by coagulation, which converts cloud drops into rain drops. The process of detrainment at the upper layers will lead to the horizontal spread of the cloud and evaporation of cloud water. The complex interplay between these processes, together with the sampling effects from G4 clouds having a wide range of cloud-top heights, complicates the simple physical explanation of the structure of PDFs in the TOGA case.

Similar to cloud water PDFs, rainwater PDFs $q_{r}$ are also positively skewed (Fig. 8), but the distributions are narrower than the cloud water PDFs. The PDF breadth increases with height $-a$ behavior that is indicative of detrainment and evaporation of rain drops. The maxima of $q_{r}$ distributions are more pronounced at the bottom and top of TOGA clouds, with somewhat flatter distributions in the middle. The shifting of the maximum rainwater frequency to the left is reflective of the fact that rain at cloud base is concentrated in narrow rain shafts and, thus, majority of cloudy pixels will have small values of $q_{r}$. The height dependence of the 1D PDFs (Figs. 6-8) extends to the 2D joint PDFs (JPDFs) (see section 4 below). 

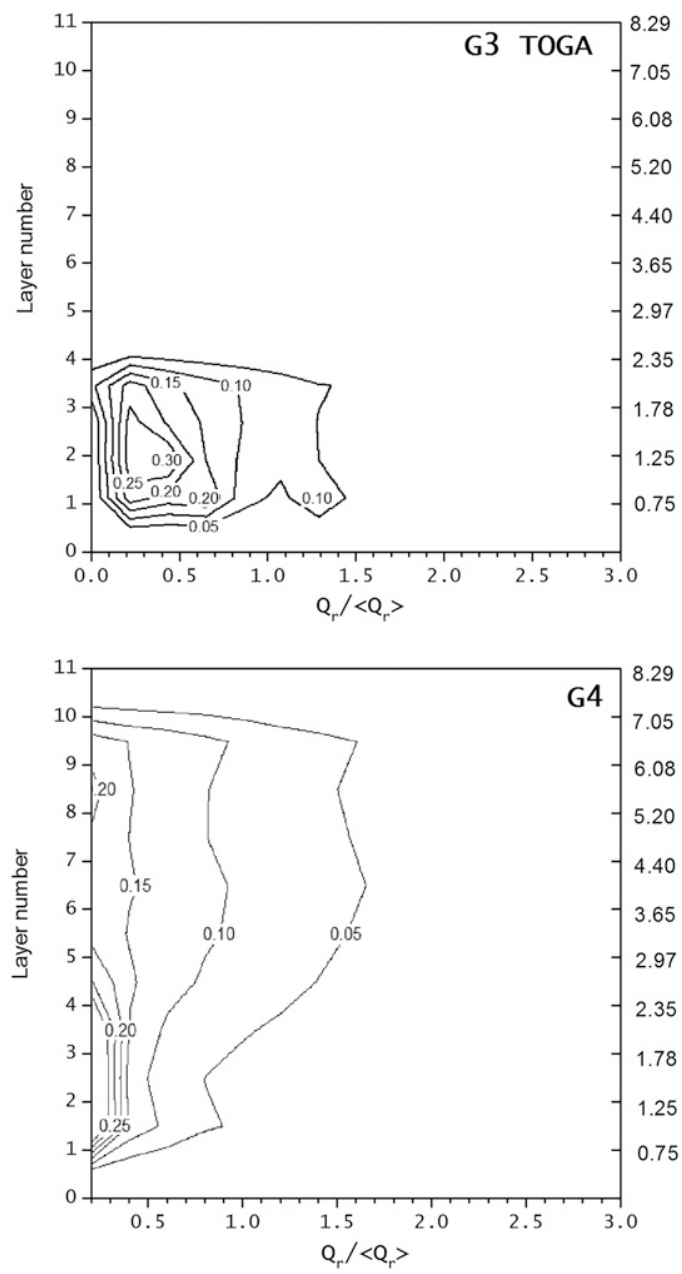
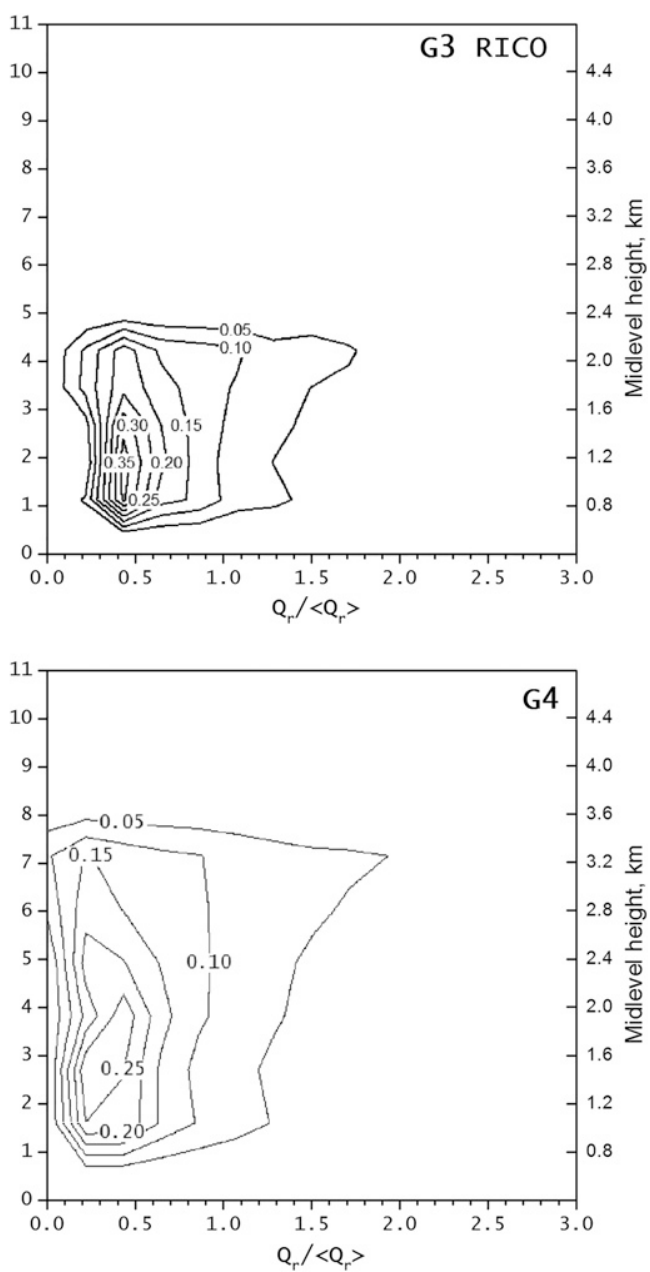

FIG. 8. As in Fig. 6, but for rainwater mixing ratio.

KM2014 demonstrated that in RICO trade cumulus an amplitude version of the Gaussian peak function (1) may represent the PDFs of cloud drop concentration:

$$
f(x)=y_{0}+A \exp \left[-\frac{\left(x-x_{c}\right)^{2}}{2 w^{2}}\right],
$$

while the lognormal distribution (2) accurately represents the cloud and rainwater PDFs

$$
f(x)=y_{0}+\frac{A}{\sqrt{2 \pi} w x} \exp \frac{-\left[\ln \left(x / x_{c}\right)\right]}{2 w^{2}} .
$$

The analysis of PDFs shape and form in the TOGA case shows that an additional analytical functional form, namely the exponential decay fit (3) may also be accurate for TOGA PDFs:

$$
f(x)=y_{0}+A \exp \left(-\frac{x}{w}\right) .
$$

Figure 9 shows examples of individual PDFs plots (solid lines) for the TOGA G4 clouds and their approximations by various fits (dashed lines). For drop concentration, the lower-layer PDFs [black line (L2) in the top panel in Fig. 9] can be well approximated by the Gaussian fit. The lognormal fit is more appropriate for the middle layers [blue line (L5)], while the most accurate approximation for the upper-layer PDFs are given the exponential decay fit (3). The parameters of the distributions are shown in Table 1 together with the calculated correlation coefficients, which are quite high for all fits $\left(R^{2}>0.95\right)$.

The noted positive skewness of the cloud and rainwater PDFs suggests the appropriateness of using a lognormal distribution, though the middle and lower panels in Fig. 9 demonstrate an exponential decay fit may be even more accurate at some levels. The exponential decay fit may most accurately represent the rainwater PDFs. The correlation coefficients for all $q_{c}$ and $q_{r}$ PDF fits are quite high $\left(R^{2}>0.98\right)$. Finally, we 

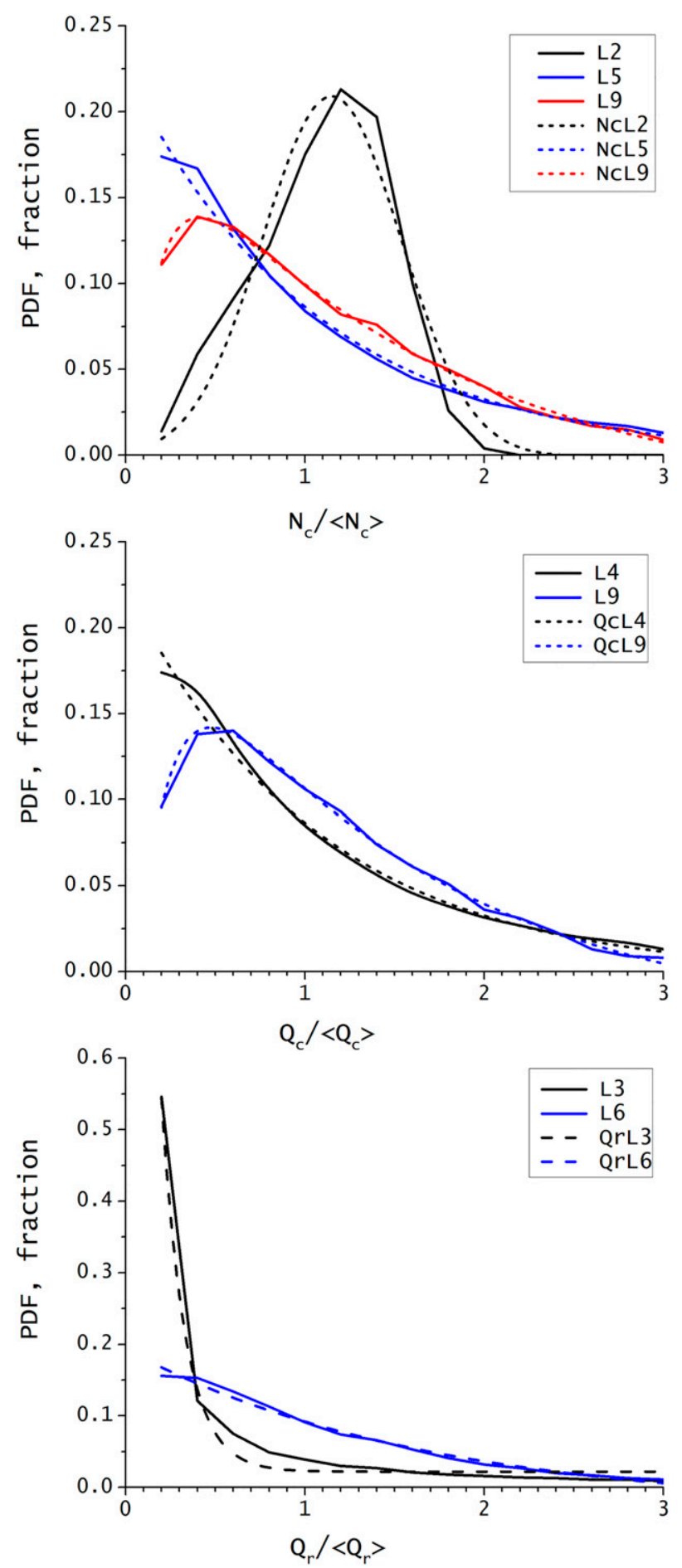

FIG. 9. Examples of PDFs of nondimensional (top) $N_{c}$, (middle) $q_{c}$, and (bottom) $q_{r}$ at indicated layers $L$. The corresponding dashed lines depict the three types of analytical fits (Gaussian, exponential decay, and lognormal) defined by (1)-(3) with parameters shown in Table 1. would like to note that analytical fits are used only for illustrating the shape of PDFs obtained from LES data. The joint PDFs that we suggest for parameterizing conversion rates are calculated using LES data and presented as height-dependent $2 \mathrm{D}$ tables with nondimensional variables discretized into 15 bins.

\section{Two-dimensional PDFs}

For the convenience of the reader, we shortly summarize the theoretical formulation of our PDF approach as described in KM2014. Microphysical process rates are typically formulated using at least two different microphysical variables. For example, the autoconversion rate may be of the form (Khairoutdinov and Kogan 2000)

$$
R_{\text {auto }}=C_{\text {au }} q_{c}^{\alpha} N_{c}^{\beta} .
$$

The integration over the grid thus should mathematically employ a JPDF to capture the mutual variability of these quantities. For this reason, we now consider the full two-dimensional PDFs of $q_{c}$ and $N_{c}$ (input variables for autoconversion) and of $q_{c}$ and $q_{r}$ (input variables for accretion) for the TOGA congestus case and compare them with the corresponding PDFs from the shallow RICO trade cumulus. We also evaluate whether the full two-dimensional PDFs can be approximated (parameterized) by the product of two one-dimensional PDFs of the individual variables.

Figure 10 shows the two-dimensional JPDF of cloud mixing ratio and cloud drop number concentration $\Omega\left(q_{c}, N_{c}\right)$ alongside the 2D PDFs calculated as a product of the two 1D PDFs of $q_{c}$ and $N_{c}$-that is, using an approximation in the form

$$
\Omega \approx f\left(q_{c}\right) g\left(N_{c}\right) .
$$

Here, functions $f\left(q_{c}\right)$ and $g\left(N_{c}\right)$ are 1D PDFs of $q_{c}$ and $N_{c}$, respectively. The joint PDFs approximated as products of the one-dimensional PDFs exhibit artifacts in the form of distinct ridges aligned parallel to the $x$ and $y$ axes. The 2D JPDFs on the left panels, on the other hand, are fully two-dimensional of the form $h(x, y)$ and show broad distributions in cloud water and narrower distributions in droplet concentration. The outcome that $h(x, y) \neq f(x) g(y)$ indicates that the two quantities covary and thus are not independent. We note that the variation of $2 \mathrm{D}$ JPDFs across different layers is greater than that of the shallower RICO clouds (Fig. 11), as indicated, for example, by the narrower distribution of $q_{c}$ and $N_{c}$ at the upper levels of the congestus clouds (cf. JPDFs for layer 6 in Figs. 10 and 11). This suggests that approximating joint $q_{c}-N_{c}$ 
TABLE 1. Best-fit parameters and errors for approximations of PDFs of $N_{c}, q_{\mathrm{c}}$, and $q_{\mathrm{r}}$. The columns $y_{0}, A, \mathrm{Xc}$, and $W$ respectively denote the offset, the amplitude, the mode, and the width of the distributions (1)-(3); $R^{2}$ is the square of correlation coefficient, also referred to as coefficient of determination.

\begin{tabular}{clcccc}
\hline \hline Curve label & \multicolumn{1}{c}{ Fit type } & $y_{0}$ & $A$ & Xc & $W$ \\
\hline NcL2 & Gauss & $-6.8 \times 10^{-4}$ & 0.21 & 1.15 & 0.39 \\
NcL5 & Exponential decay & -0.002 & 0.23 & - & 1.065 \\
NcL9 & Lognormal & -0.057 & 0.66 & 2.46 & 1.34 \\
QcL4 & Exponential decay & -0.002 & 0.23 & - & 1.06 \\
QcL9 & Lognormal & -0.050 & 0.52 & 1.82 & 1.16 \\
QrL3 & Exponential decay & 0.022 & 2.37 & - & 0.99 \\
QrL6 & Exponential decay & -0.031 & 0.22 & - & 0.99 \\
\end{tabular}

distributions by a single, height-independent JPDF may be significantly less accurate for congestus clouds than for the shallow cumulus clouds from the RICO case. Therefore, such a fixed-height approximation will not be considered here, and we will instead concentrate on evaluating the accuracy of JPDFs calculated for each of the 10 vertical layers.

The shapes of the fully two-dimensional JPDFs of $q_{c}$ and $q_{r}$ (the quantities used to calculate accretion in bulk models) in Fig. 12 differ substantially from the PDFs of $q_{c}$ and $N_{c}$. The rainwater mixing ratio distribution varies substantially with height - a result consistent across both congestus and trade cumulus simulations (Figs. 12 and 13). At the lower levels, the JPDFs seem to closely resemble the 2D PDFs represented by a product of the individual 1D PDFs. This, similar to the RICO case, reflects a weaker correlation between $q_{c}$ and $q_{r}$ than between $q_{c}$ and $N_{c}$, which seems to be contradictory to the fact that taller clouds contain more cloud water and also produce more precipitation. While $q_{c}$ and $q_{r}$ may indeed correlate on the scale of the entire cloud or cloud system [see, e.g., Lebsock et al. (2013), who found a robust correlation between cloud and rainwater based on CloudSat data], over small LES grid volumes $q_{c}$ and $q_{r}$ may spatially decorrelate. The issue of scale as to the correlation or noncorrelation of $q_{c}$ and $q_{r}$ is discussed in detail in KM2014.

In summary, PDFs of trade cumulus and congestus show much similarity, except that the TOGA PDFs exhibit greater height dependence and a greater difference between the JPDFs and the PDFs approximated by the product of $1 \mathrm{D}$ individual PDFs. In section 5, we evaluate layer-mean microphysical process rates using both the "full" JPDFs and the 2D PDFs approximated by the product of the two 1D PDFs according to (5). We find that the difference between the two representations of the JPDFs is pronounced for the $q_{c}$ and $N_{c}$, which, along with the substantial nonlinearity of the autoconversion rate, results in a substantial impact on the layer-averaged autoconversion rates. Compared to autoconversion, the accretion rate, which employs the JPDF of $q_{c}$ and $q_{r}$, is less sensitive to details about how the PDF is formulated.

\section{Testing of the PDF parameterization}

Similar to KM2014, we introduce dimensionless parameters $\phi$ and $\psi$ defined as

$$
\varphi=q_{c} / \overline{q_{c}}, \quad \psi=N_{c} / \overline{N_{c}}, \quad \zeta=q_{r} / \overline{q_{r}},
$$

where $\overline{q_{c}}, \overline{N_{c}}$, and $\overline{q_{r}}$ are the grid-volume mean values of $q_{c}, q_{r}, N_{c}$ taken over the cloudy areas of each layer. Unbiased grid-mean process rates are calculated by integrating the local process rate over the JPDF. For autoconversion, this becomes

$$
\begin{aligned}
\bar{R}_{\text {auto }} & =\overline{C_{\text {au }} q_{c}^{\alpha} N_{c}^{\beta}} \equiv \iint C_{\text {au }} q_{c}^{\alpha} N_{c}^{\beta} \Omega\left(q_{c}, N_{c}\right) d q_{c} d N_{c} \\
& =\left.C_{\text {au }}\left(\overline{q_{c}}\right)^{\alpha}\left(\overline{N_{c}}\right)^{\beta} \iint \varphi^{\alpha} \psi^{\beta} \Omega^{\prime}\right|_{\overline{q_{c}} \overline{N_{c}}}(\varphi, \psi) d \varphi d \psi .
\end{aligned}
$$

As in KM2014, $\Omega$ is the JPDF of $q_{c}$ and $N_{c}$, and $\Omega^{\prime}$ is its nondimensional analog transformed from $q_{c}$ and $N_{c}$ via the relationship

$$
\Omega^{\prime}(\varphi, \psi) d \varphi d \psi=\Omega\left(q_{c}, N_{c}\right) d q_{c} d N_{c} .
$$

We can then define a multiplicative factor $D_{\mathrm{au}}$, which consolidates the effects of subgrid-scale variability on the microphysical process rate:

$$
D_{\mathrm{au}}\left(\overline{q_{c}}, \overline{N_{c}}\right)=\left.\iint \varphi^{\alpha} \psi^{\beta} \Omega^{\prime}\right|_{\overline{q_{c}} \overline{N_{c}}}(\varphi, \psi) d \varphi d \psi
$$

Using (9), we can rewrite (7) as

$$
\bar{R}_{\text {auto }}=C_{\text {au }}\left(\overline{q_{c}}\right)^{\alpha}\left(\overline{N_{c}}\right)^{\beta} D_{\text {au }}\left(\overline{q_{c}}, \overline{N_{c}}\right) .
$$

Note that the factor $D_{\text {au }}$ convolves both the subgridscale variability and the nonlinearity of the process rate. In the case when $D_{\text {au }}[(9)]$ is calculated using the $2 \mathrm{D}$ 


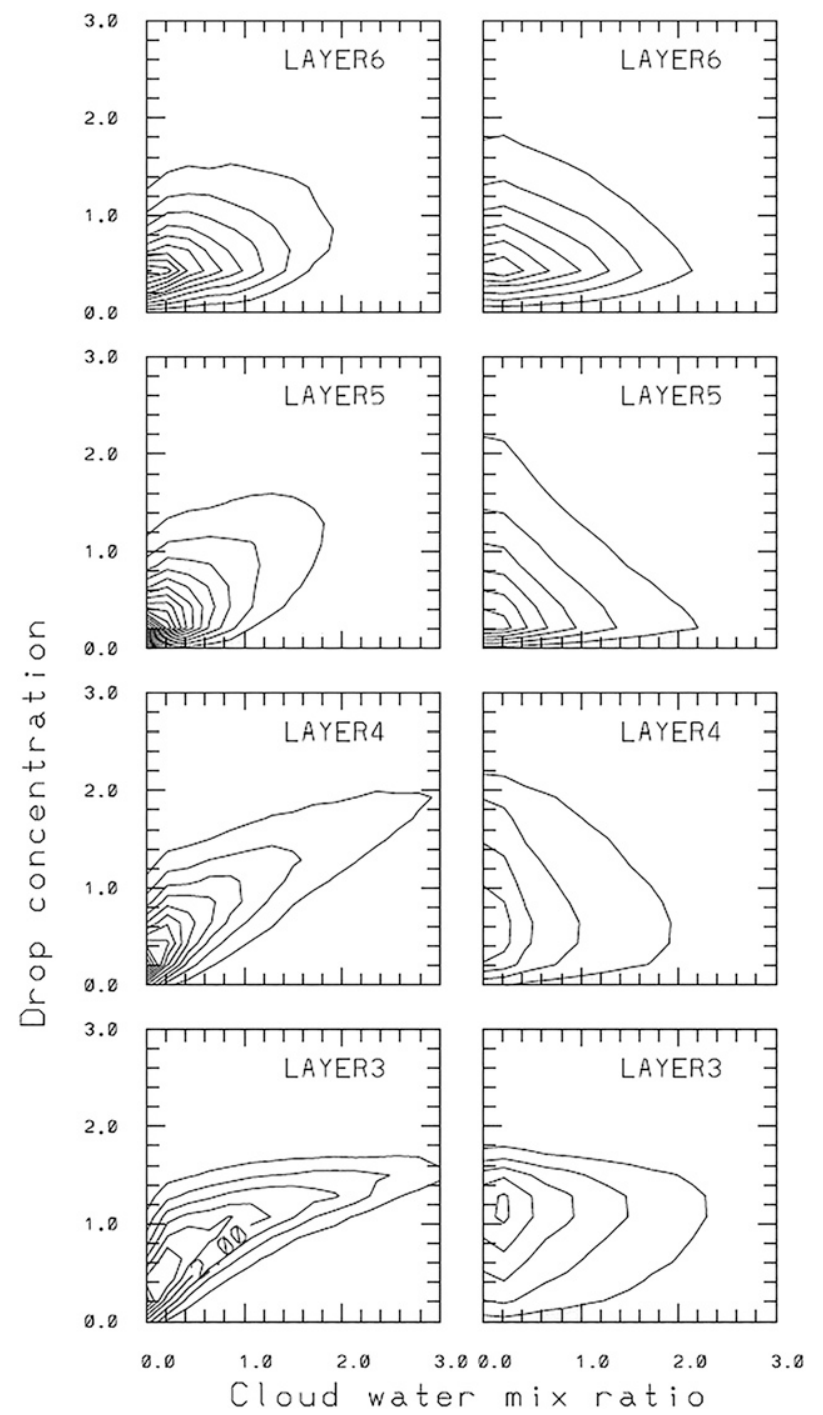

FIG. 10. Isolines of the PDF parameters $q_{c}$ and $N_{c}(\%)$ at indicated layers for the TOGA simulation. (left) The "exact" JPDF and (right) the 2D PDF as a product of 1D individual PDFs of $q_{c}$ and $N_{c}$. This example is for group 4 based on the 36-72-hourly data output. Contour interval is 0.5 .

JPDF $\Omega$, the resulting autoconversion rates are referred to as "au2D" in the plots below. In the case when the factor $D_{\text {au }}$ employs approximation of $\Omega$ by the product of 1D functions $f\left(q_{c}\right)$ and $g\left(N_{c}\right)(5)$, the calculated autoconversion rates are referred to as "au1D."

The local autoconversion rate is a highly nonlinear function of variables $q_{c}$ and $N_{c}$, so we can expect substantial difference between the exact expression given by (10) and the approximation simply based on grid averaged parameters, which neglects the subgrid-scale variability and the resulting PDF enhancement factor $D_{\text {au: }}$ :

$$
\bar{R}_{\text {auto }}^{\text {mean }} \approx C_{\text {au }}\left(\overline{q_{c}}\right)^{\alpha}\left(\overline{N_{c}}\right)^{\beta} .
$$

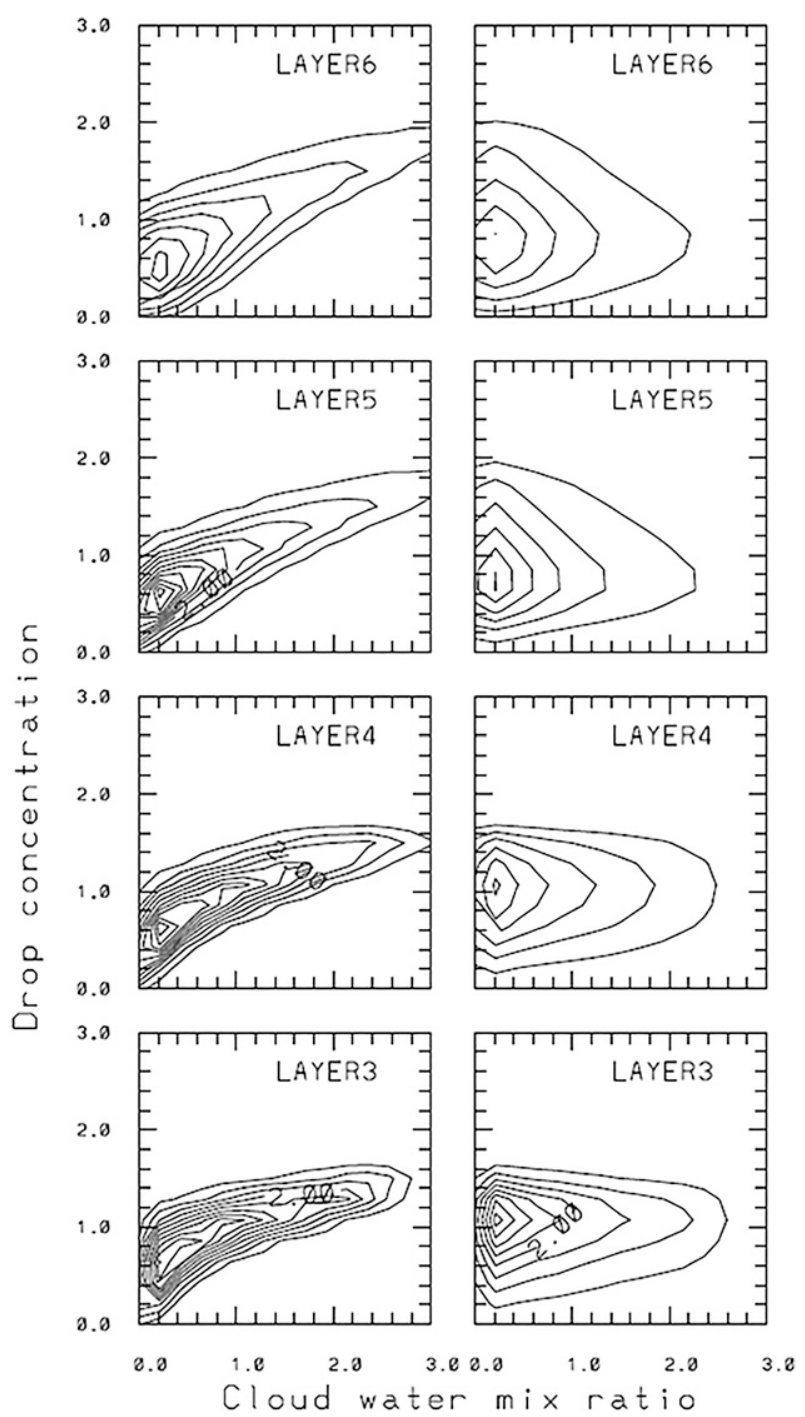

FIG. 11. As in Fig. 10, but for the RICO simulation.

The approximation (11) is referred to as "auMean" in the plots shown below.

All three PDF formulations are evaluated by comparing them to the benchmark ("true") rates. The latter are obtained by averaging the local conversion rates at each LES model grid point $(i, j)$ over each horizontal layer:

$$
\bar{R}_{\text {auto }}^{\text {true }}=\sum_{i, j} C_{\text {au }} q_{c}^{\alpha}(i, j) N_{c}^{\beta}(i, j) / M
$$

where $M$ is the total number of cloudy pixels in the layer $L$ at time $t$. The calculations, one for autoconversion (12) and the similar one for accretion, were performed for each layer $L$, each archived time step $t$, and each of the three cloud groups (group G1 is not considered owing to negligible contribution to precipitation). These 


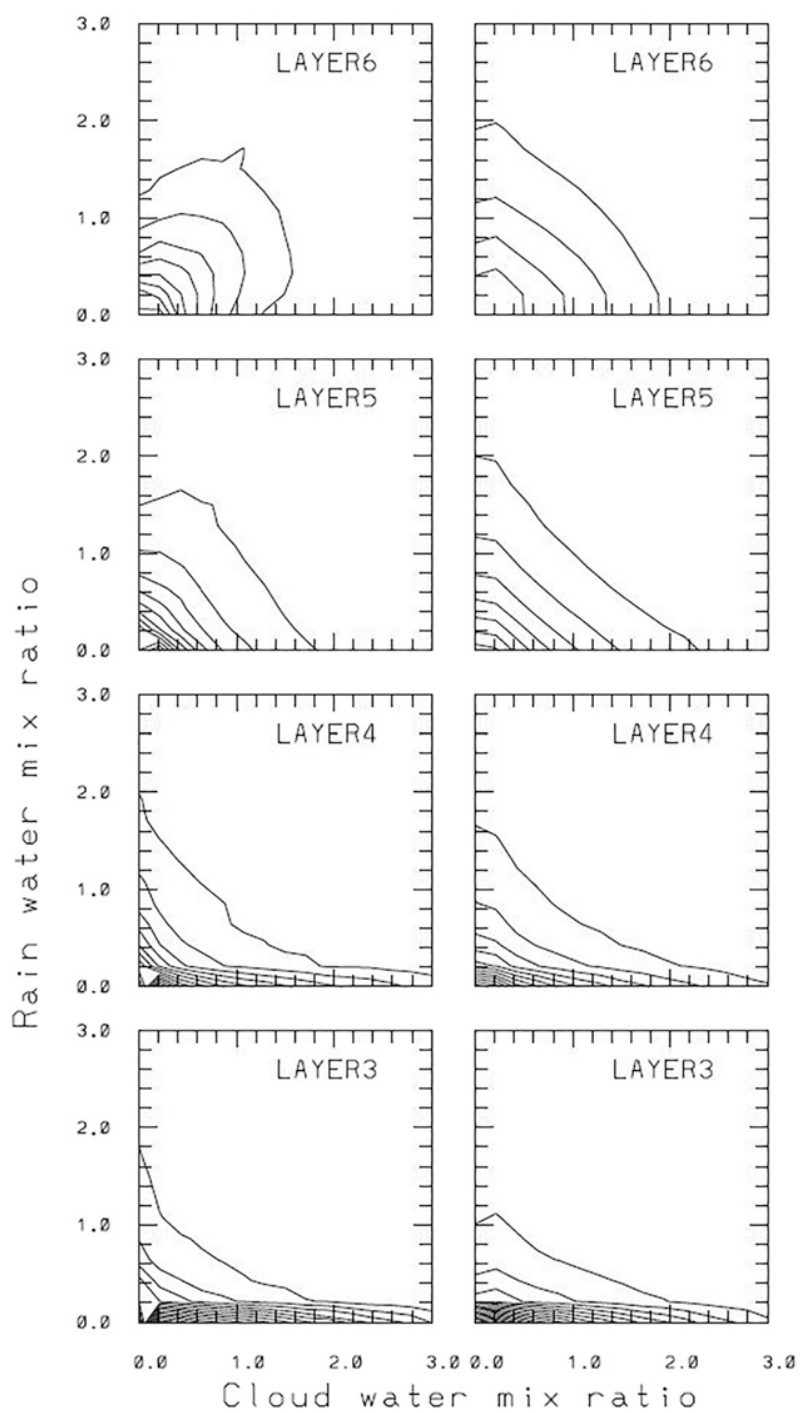

FIG. 12. As in Fig. 10, but for parameters $q_{c}$ and $q_{r}$.

formulations establish the benchmark (true) process rates. Each analysis layer consists of $384 \times 384 \times 10$ points, but because of the small cloud fraction in the TOGA case $(\sim 0.1)$, the number of cloudy points in each layer is only about $2 \times 10^{5}$. Applying this procedure for each hourly simulation output at each layer and cloud group resulted in about 700 benchmark measures of layer-mean process rates. The same procedure was applied for the evaluation of accretion rates where instead of variables $q_{c}$ and $N_{c}$ the pair of variables $q_{c}$ and $q_{r}$ has been used.

The parameterized conversion rates are evaluated for each of the following approaches: 1) based on the 2D JPDF, 2) based on the 2D PDF approximated by a product of two 1D PDFs, and 3) based only on the layermean variables $q_{c}, N_{c}$, and $q_{r}$. While the first two

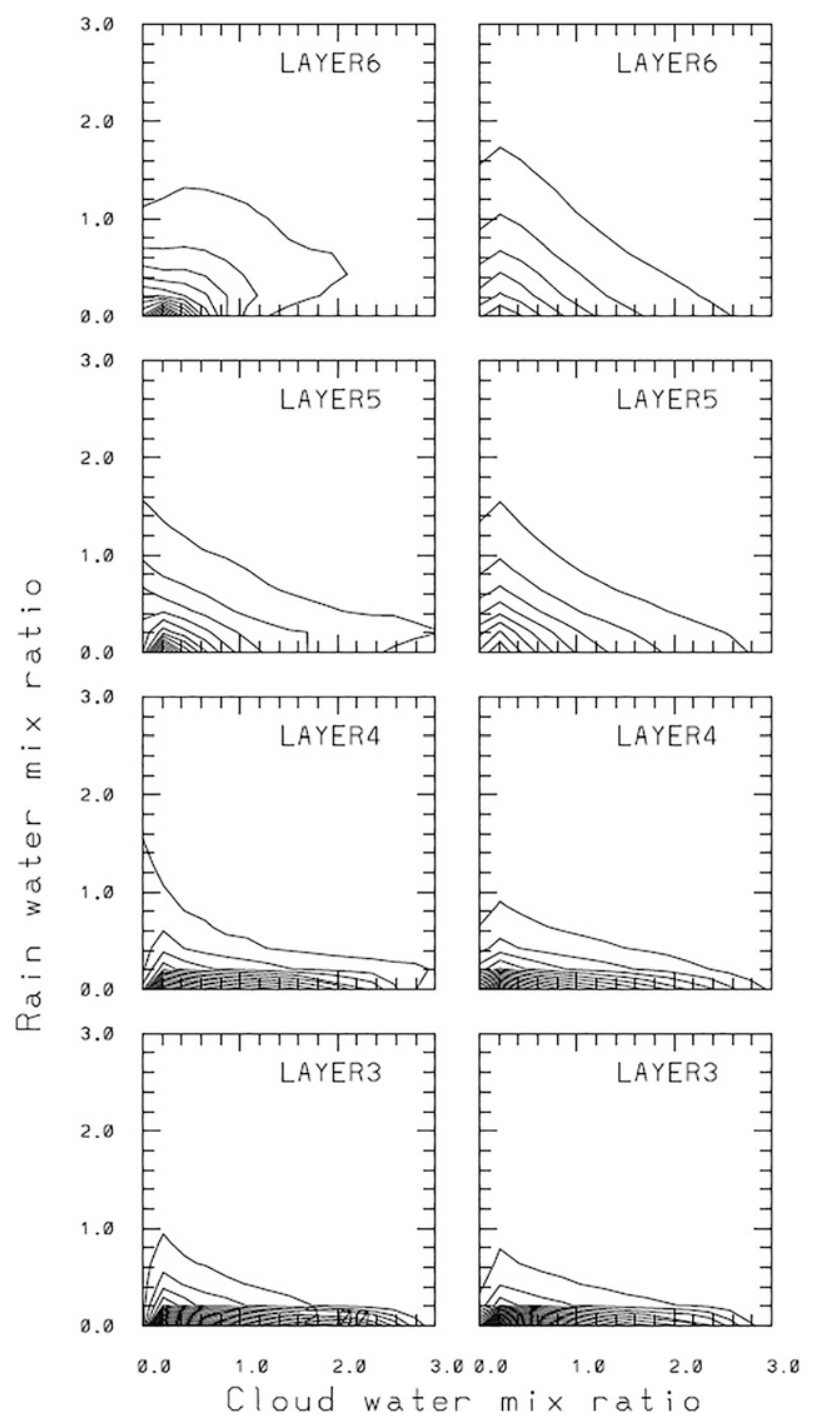

FIG. 13. As in Fig. 11, but for parameters $q_{c}$ and $q_{r}$.

calculations employ different PDF formulations for obtaining grid-averaged conversion rates, the third calculation completely neglects SGS variability. Even in the most accurate approach (number 1), we need to make certain assumptions and simplifications consistent with a possible implementation of the PDF approach in mesoscale models. In the ideal case, we would use PDFs, which are time and cloud group dependent-that is, obtained using the hourly datasets for each of the three groups. Such time- and group-dependent PDFs would significantly complicate the formulation of PDFs and their implementation in mesoscale models, however. The much simpler formulation is to use fixed-in-time JPDFs obtained from the entire dataset over the entire simulation period for the group that contributes most to precipitation-in the case of TOGA, the group G4 

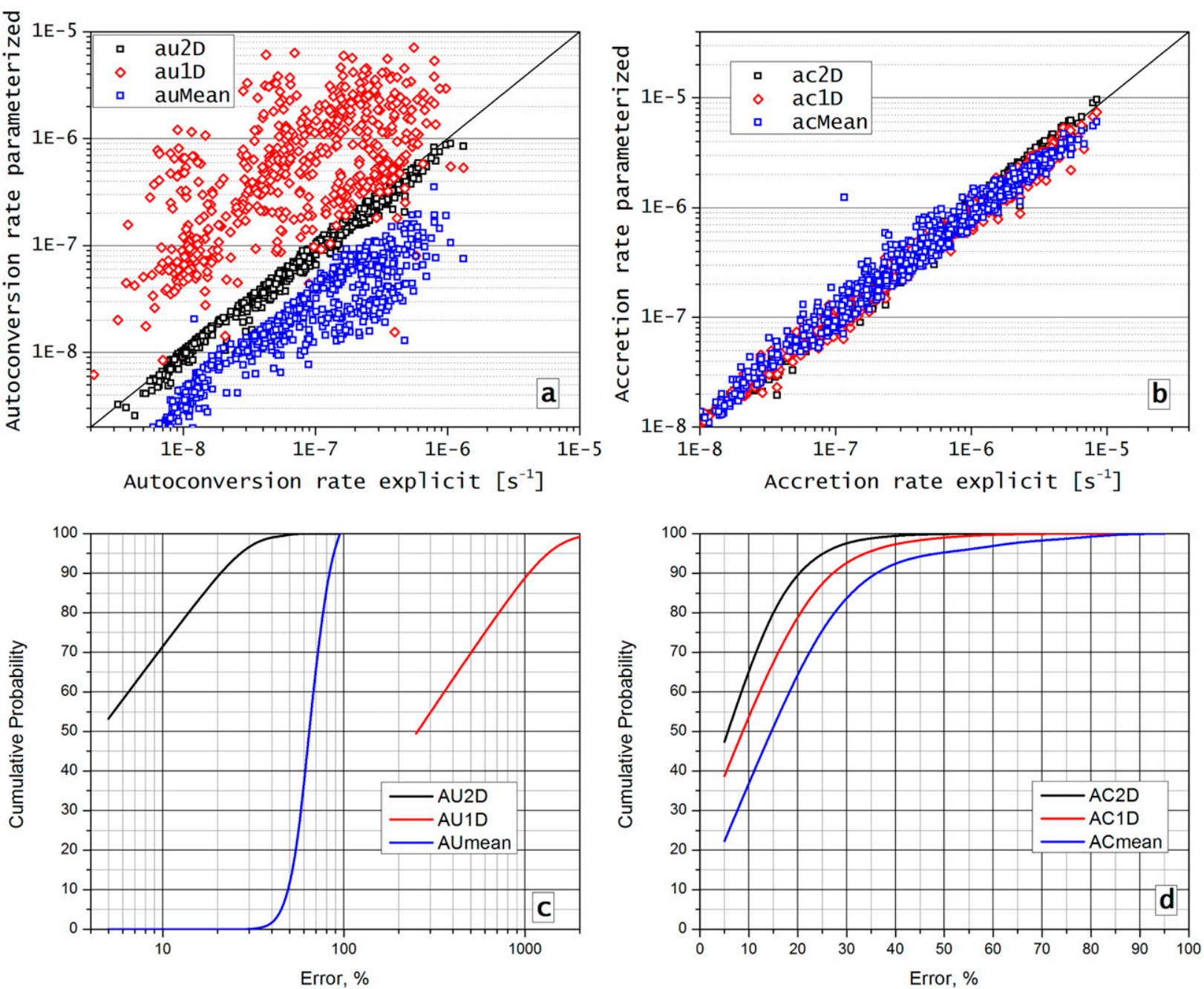

FIG. 14. Comparison between conversion rates $\left(\mathrm{s}^{-1}\right)$ using different formulations of the PDF. Black squares are JPDFs of $q_{c}$ and $N_{c}$ in the case of autoconversion rate and $q_{c}$ and $q_{r}$ in the case of accretion rate. Red diamonds are PDFs based on the product of two separate 1D distributions. Blue squares are conversion rates calculated without the use of a PDF based on grid-mean values of corresponding parameters.

comprises the tallest clouds. The tests made in KM2014 showed that the use of such time-independent PDFs only slightly decreases the accuracy of layer-mean conversion rates. As we show below, in the TOGA case the use of such a simplified approximation degrades the accuracy of layer-mean conversion rates more significantly. Nevertheless, a time-independent PDF approach is still a substantial improvement compared to neglecting subgrid variability and using only the layer-mean variables to calculate microphysical process rates.

Figure 14 shows the comparison of autoconversion and accretion process rates calculated using these three approaches. The top panels show scatterplots for the different PDF formulations, while the bottom panels show cumulative distributions of errors. More accurately accounting for the variability using the time- and groupdependent JPDF results in the most accurate values for both autoconversion and accretion rates (au2D and ac2D). For autoconversion rates, the mean absolute relative error is $11.8 \%$, with the standard deviation of the absolute relative error being $10.9 \%$; for accretion rates, the mean error is $12.3 \%$ with standard deviation of $9.7 \%$. The maximum errors are less than $40 \%$ for both autoconversion and accretion rates.

Approximating the JPDFs as the product of 1D PDFs (au1D and ac1D) substantially overestimates the autoconversion rates: for example, about $10 \%$ of the autoconversion rates exhibit errors of at least $1000 \%$ (Fig. 14c). This substantial overestimate in autoconversion results directly from the 1D PDF method 
overestimating the frequency of low drop concentrations (e.g., layer3 in Fig. 10). Neglecting subgrid variability completely (auMean and acMean), on the contrary, produces the classic underestimate of autoconversion rate (Fig. 14a) resulting in errors between $50 \%$ and $90 \%$.

Accretion rate errors $(\sim 40 \%-50 \%)$ are much smaller than autoconversion errors (Figs. 14b,d) because of the weaker correlation between $q_{c}$ and $q_{r}$ and because of the weaker nonlinearity of the accretion rate formula. Errors increase when approximating the JPDF as the product of two 1D PDFs and can reach $60 \%-80 \%$, although for $95 \%$ of the points the error is less than $35 \%$ (Fig. 14d). Completely neglecting SGS variability leads to further increase in errors; however, they are still significantly less than autoconversion errors. Neglecting variability overestimates small and medium accretion rates and underestimates large rates (Fig. 14b).

While the use of time- and group-dependent JPDF will result in the most accurate values for both conversion rates, such a formulation where the shape of a PDF is dependent on time (hence layer-mean variables) and on cloud group (i.e., cloud-top height) is difficult to obtain. In addition, the subgrid information on cloudtop heights inside a mesoscale model grid is in principle not available. We therefore test feasibility of two simplified approximations, both using JPDFs obtained for the whole dataset that do not depend on layer-mean variables. In the first test, we use separate JPDFs for each of the three cloud groups (AU2DvarG; magenta curve), while in the second test (AU2DfxG4; red curve) we use only one JPDF derived for G4 clouds (i.e., with tops higher than $2.7 \mathrm{~km}$ ). Figure 15 shows cumulative errors of such simplified approximations. For reference, we also repeat previously shown in Fig. 14 curves for the most accurate, time- and group-dependent formulation (AU2D; black curve) and the formulation neglecting SGS variability using only layer-mean variables (blue curve; AUmean). The simplified formulations substantially degrade the accuracy: the case AU2DvarG for autoconversion rate has mean errors of $21.4 \% \pm 25.8 \%$, while the case AC2DvarG for accretion rate has mean errors of $14.4 \% \pm 10.4 \%$. A further simplification using the JPDFs from only the G4 group results in even larger errors, though the additional increase in error is less dramatic than that exhibited by AU2DvarG and AC2DvarG. In the case AU2DfxG4, the autoconversion rate mean errors are $26.6 \% \pm 27.8 \%$, while in the case AC2DfxG4 the accretion rate mean errors are $17.4 \% \pm 11.2 \%$. For comparison, the true formulations AU2D and AC2D have mean errors on the order of $12 \%$, and the cases AUmean and ACmean have mean errors of $73.8 \% \pm 10.7 \%$ and $21.9 \% \pm 17.8 \%$,
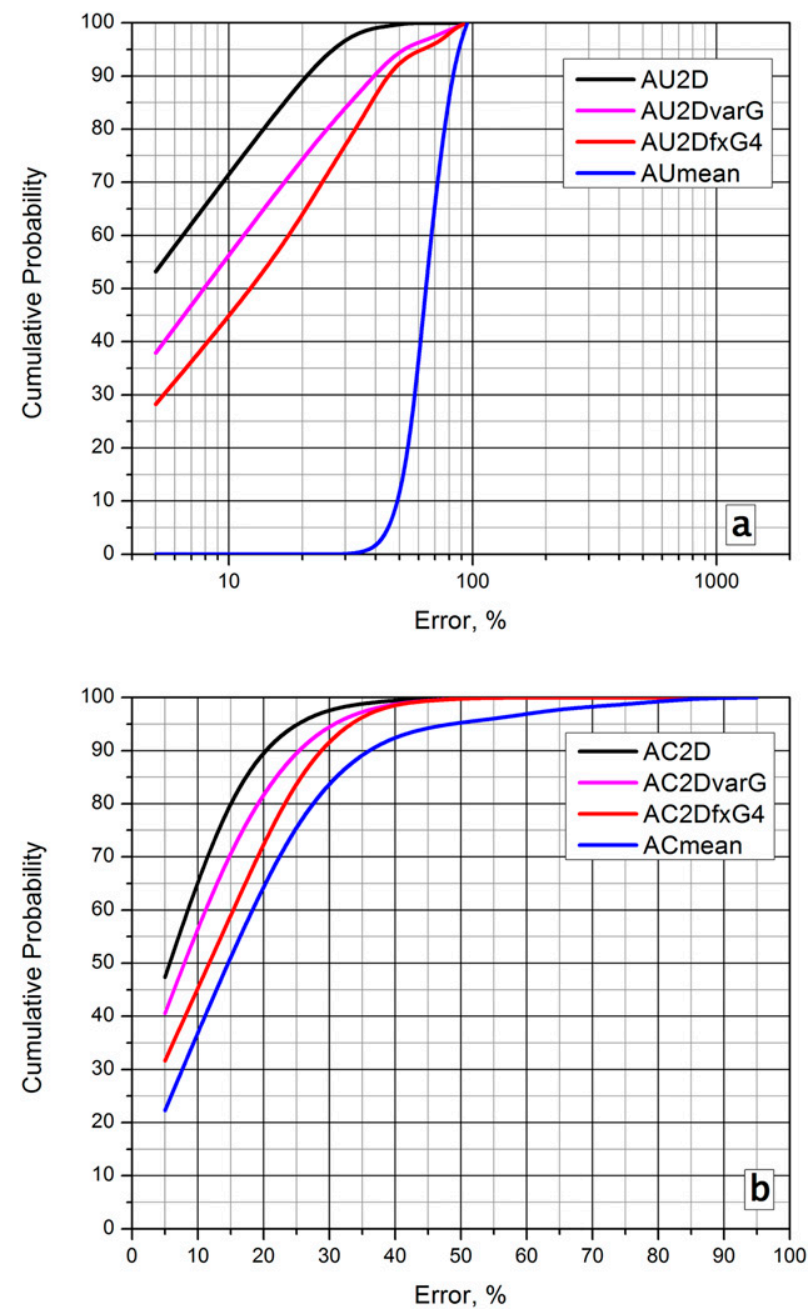

FIG. 15. Cumulative distribution of errors in (a) autoconversion and (b) accretion rates calculated using various approximations for 2D JPDFs. The black line corresponds to the most accurate timeand height-dependent JPDF; the magenta line corresponds to a fixed-in-time but height-dependent JPDF; the red line corresponds to a JPDF fixed in time and obtained for clouds in the G4 group only. For comparison, the blue line shows errors in a case when only layermean variables are accounted and no PDFs are applied.

respectively. In summary, we can conclude that even using highly simplified formulations of JPDFs reduce the errors compared to the case when SGS variability is neglected altogether.

Because the TOGA simulation includes a wide variety of clouds, not only congestus but also shallow cumulus as well, we were curious about the generality of our PDFs [addressed also by Bogenschutz et al. (2010)]. A premise of the generality of the TOGA PDFs is suggested by the fact that the TOGA dataset covers not a different set of parameters but a wider set of parameters (see Fig. 3). In other words, are the TOGA PDFs suitable to be universally applied to both shallow and 


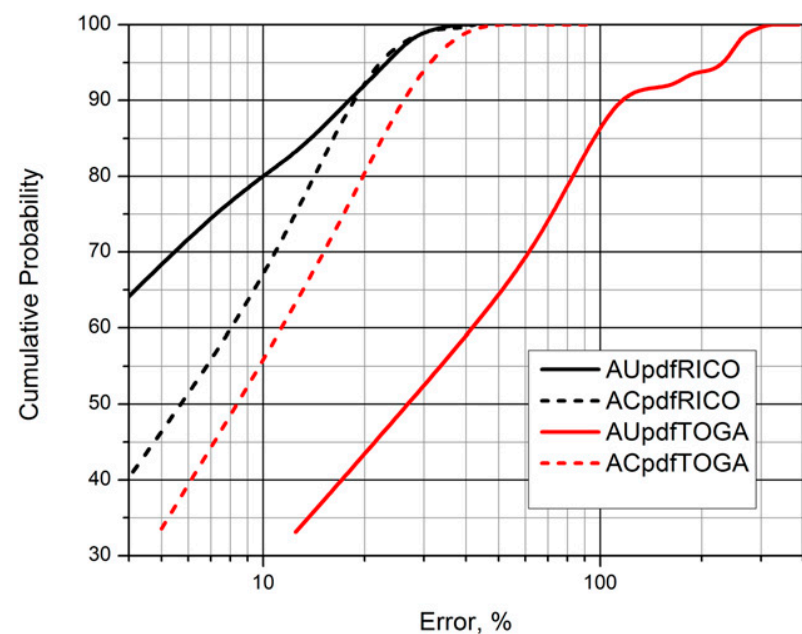

FIG. 16. Cumulative distribution of errors in autoconversion (solid lines) and accretion (dashed lines) rates calculated using 2D JPDFs obtained based on RICO (black) and TOGA (red) datasets.

congestus cloud types? To answer this question, we evaluated process-rate errors in the RICO simulation using PDFs obtained from the TOGA dataset. The black lines in Fig. 16 quantify the process-rate error using the "native" RICO PDFs in the RICO simulations, and the red lines represent the error applying the TOGA PDFs to the RICO simulations. These results indicate that the autoconversion rates are highly sensitive to which dataset is used, and the misapplication of the TOGA PDFs results in an increase in autoconversion rate errors by an order of magnitude (from $30 \%$ maximum to $300 \%$ maximum error). The accretion rate errors depend significantly less on which PDFs are employed, whether from the TOGA or RICO datasets, although using the "foreign" PDF nevertheless increases the error from about $22 \%$ to $30 \%$ (the $95 \%$ cumulative error value).

\section{Conclusions}

Calculating unbiased microphysical process rates over mesoscale model grid volumes necessitates knowledge of the subgrid-scale distribution of variables, typically represented as PDFs of the different variables. Microphysical process rates integrated over these PDFs represent exact averages of the process rates across a mesoscale grid volume. KM2014 employed large-eddy simulation of RICO trade cumulus to develop 1D PDFs of $q_{c}, N_{c}$, and $q_{r}$, as well as joint PDFs (JPDF) of pairs of variables, $\left(q_{c}\right.$ and $\left.N_{c}\right)$ and $\left(q_{c}\right.$ and $\left.q_{r}\right)$, to be used in the autoconversion and accretion process-rate formulas.

While KM2014 considered shallow trade wind cumulus clouds, observations show that cumulus congestus constitute an important component of tropical cloudiness. Congestus contribute to the tropical precipitation and diabatic heating and may serve to moisten and destabilize the tropical atmosphere, thus possibly playing a role in MJO transitions and the large-scale tropical circulation. We have extended the method of KM2014 to a population of deeper precipitating cumulus congestus clouds and developed and evaluated the accuracy and generality of a hierarchy of JPDF formulations using TOGA datasets.

The following findings of the KM2014 (employed in this study as well) were essential for formulation of JPDFs. First, and perhaps the most important, was the decision to express the PDF as a function of nondimensional variables obtained by normalizing physical variables by their layer-mean values. The analysis of PDFs in the dimensional physical space proved to be unsuccessful, as these PDFs exhibit substantial spatial variation with no easily discernable patterns or regularity. Their nondimensional counterparts, however, reveal more stable and identifiable physical dependencies. Second, the statistical robustness of PDFs was facilitated by the decision to formulate PDFs in layers containing multiple vertical levels (10 levels per layer in the RICO and TOGA cases). And finally, the use of LESs for providing the 3D fully integrated datasets was essential for formulating and evaluating the PDFs.

The JPDFs obtained from statistical analysis of LES datasets were formulated as a set of tables dependent on the vertical layer (see electronic supplement). Each JPDF of a pair of variables can be integrated to obtain a process-rate multiplicative PDF enhancement factor that is a function of the layer-mean microphysical variables. This approach has an advantage over analytical formulation of PDF if such formulation would be possible; namely, a formal integration (either analytic or numerical) to obtain PDF is not required every time a process rate is calculated.

When applying the JPDF in a mesoscale model, one needs to account not only for its variability in the vertical but also for its dependence on mesoscale resolvable variables. In our formulation, a mesoscale grid is represented by our entire integration domain, and layermean variables of our domain correspond to resolvable prognostic variables of the mesoscale model. As a simulation evolves, these layer-mean variables change, and the dependence of the shape of the JPDFs on layermean variables may be evaluated by comparing JPDFs obtained from hourly datasets with the fixed-in-time JPDFs obtained using the entire simulation dataset. The JPDFs also depend on cloud-top height, and hence they vary from one cloud group to another. 
Clearly, the use of time- and group-dependent JPDF proved to provide the most accurate conversion rates. However, formulating the temporal dependence of the shape of the JPDFs (or equivalently, on layer-mean variables) is quite challenging, at least at this initial stage of the PDF method development. In addition, the subgrid information on cloud-top heights inside a mesoscale model grid is unavailable.

We attempted to quantify errors in microphysical conversion rate estimates associated with a hierarchy of JPDF formulations. To this end, we tested two simplified approximations of the JPDF, both of which accounted for variation of the JPDF's shape in the vertical but neglected dependence on layer-mean variables (i.e., the time dependence). It was concluded that neglecting dependence of JPDF shape on layer-mean variables substantially degrades the accuracy of conversion rate estimates. Using only a single JPDF based on G4 clouds (i.e., with tops higher than $2.7 \mathrm{~km}$ ) increases the errors, but to a lesser degree. The increase in errors is quantified as follows: in the case of "full" JPDF formulation (accounting for the time and height dependence of the JPDFs), the mean errors are about $12 \%$ and $12 \%$ for autoconversion and accretion rate, respectively, in the case of neglecting the time dependence of the JPDFs, the mean errors are about $21 \%$ and $14 \%$, and in the case of neglecting both the time and the height dependence, the mean errors are approximately $27 \%$ and $17 \%$. These errors are still smaller compared to the case when subgrid variability is neglected altogether ( $74 \%$ and $22 \%$ ). It is worth noting that our evaluations were done in a rather large, $38 \times 38 \mathrm{~km}^{2}$ domain where clouds exhibit a wide range of depths. We surmise that the subgrid effect of varying cloud depths may diminish when smaller grids are used in mesoscale models, and therefore, PDFs for such smaller grid will characterize a narrower range of cloud depths. In summary, our tests demonstrate the degree to which various PDF formulations may improve accuracy of conversion rate estimation.

Although the new congestus PDFs are qualitatively similar to those found in KM2014 for RICO trade cumulus, nevertheless there are significant quantitative differences. As in the RICO case, the shape of individual TOGA PDFs in each layer may be approximated either by Gaussian or lognormal distributions. However, for certain layers in the TOGA case, a more accurate fit can be provided by the exponential decay function. The representation of JPDFs as the product of the 1D PDFs is inaccurate not only in the case of the JPDF of $q_{c}$ and $N_{c}$ where correlation between of $q_{c}$ and $N_{c}$ is quite strong but also in the case of the JPDF of $q_{c}$ and $q_{r}$. Contrary to results from the RICO simulation, the correlation between $q_{c}$ and $q_{r}$ in the TOGA case is much stronger, especially in the upper layers.

The TOGA simulation contains a broad spectrum of cloud depths ranging from shallow trade cumulus up to precipitating cumulus congestus, so we hoped that the PDF parameterization developed from the TOGA simulation would apply not only to congestus clouds, but also to the shallower trade cumulus. We find that the TOGA PDFs can be successfully employed for accretion rates calculations for both cloud types; however, the errors in autoconversion rates when applying the TOGA PDFs to the RICO simulations were unacceptably large. Unfortunately, this leads us to conclude that a "universal" PDF formulation for both shallow and congestus clouds may not be possible, which is unfortunate given the appeal of unified physical parameterizations and the simplicity of their implementation. We surmise that future "smart" mesoscale models will be able to diagnose the cloud-type system at each grid point based on criteria using stratification and instability (e.g., CAPE) parameters and, thus, determine which PDF formulation to apply at a particular location.

Our future work will include the implementation of a PDF parameterization in the ONR COAMPS model and evaluating the role of large errors in autoconversion, as well as smaller but still significant errors in accretion, arising from neglecting SGS variability or simplifications in PDF formulation. These future efforts will address the consequences of the status quo approach of neglecting SGS variability and quantify how much improvement can be expected by employing these new methods. Obviously, a PDF scheme may be expected to yield the greatest improvement for highly heterogeneous cloud fields.

Acknowledgments. The authors are grateful to three anonymous reviewers for many constructive comments. This investigation was supported by NOAA/Office of Oceanic and Atmospheric Research under NOAAUniversity of Oklahoma Cooperative Agreement NA17RJ1227 and NA08OAR4320904, U.S. Department of Commerce, and by ONR Grants N00014-11-10439 and N00014-11-1-0518. The computing for this project was performed at the OU Supercomputing Center for Education and Research (OSCER) at the University of Oklahoma.

\section{REFERENCES}

Beard, K. V., and H. T. Ochs, 1993: Warm rain initiation: An overview of microphysical mechanisms. J. Appl. Meteor., 32, 608-625, doi:10.1175/1520-0450(1993)032<0608: WRIAOO $>2.0 . \mathrm{CO} ; 2$. 
Bogenschutz, P. A., S. K. Krueger, and M. Khairoutdinov, 2010: Assumed probability density functions for shallow and deep convection. J. Adv. Model. Earth Syst., 2 (10), doi:10.3894/ JAMES.2010.2.10.

Casey, S. P. F., A. E. Dessler, and C. Schumacher, 2007: Frequency of tropical precipitating clouds as observed by the Tropical Rainfall Measuring Mission Precipitation Radar and ICE Sat/ Geoscience Laser Altimeter System. J. Geophys. Res., 112, D14215, doi:10.1029/2007JD008468.

—, E. J. Fetzer, and B. H. Kahn, 2012: Revised identification of tropical oceanic cumulus congestus as viewed by CloudSat. Atmos. Chem. Phys., 12, 1587-1595, doi:10.5194/ acp-12-1587-2012.

Cheng, C.-P., and R. A. Houze Jr., 1979: The distribution of convective and mesoscale precipitation in GATE radar echo patterns. Mon. Wea. Rev., 107, 1370-1381, doi:10.1175/ 1520-0493(1979)107<1370:TDOCAM >2.0.CO;2.

Comstock, K. M., C. S. Bretherton, and S. E. Yuter, 2005: Mesoscale variability and drizzle in southeast Pacific stratocumulus. J. Atmos. Sci., 62, 3792-3807, doi:10.1175/ JAS3567.1.

Godfrey, J. S., R. A. Houze Jr., R. H. Johnson, R. Lukas, J.-L. Redelsperger, A. Sumi, and R. Weller, 1998: Coupled OceanAtmosphere Response Experiment (COARE): An interim report. J. Geophys. Res., 103, 14395-14450, doi:10.1029/ 97JC03120.

Golaz, J.-C., V. E. Larson, and W. R. Cotton, 2002: A PDF-based model for boundary layer clouds. Part I: Method and model description. J. Atmos. Sci., 59, 3540-3551, doi:10.1175/ 1520-0469(2002)059<3540:APBMFB > 2.0.CO;2.

Hohenegger, C., and B. Stevens, 2013: Preconditioning deep convection with cumulus congestus. J. Atmos. Sci., 70, 448-464, doi:10.1175/JAS-D-12-089.1.

Hollars, S., Q. Fu, J. Comstock, and T. Ackerman, 2004: Comparison of cloud-top height retrievals from ground-based $35 \mathrm{GHz}$ MMCR and GMS-5 satellite observations at ARM TWP Manus site. Atmos. Res., 72, 169-186, doi:10.1016/ j.atmosres.2004.03.015.

Jensen, M. P., and A. D. Del Genio, 2006: Factors limiting convective cloud-top height at the ARM Nauru island climate research facility. J. Climate, 19, 2105-2117, doi:10.1175/ JCLI3722.1.

Johnson, R. H., T. M. Rickenbach, S. A. Rutledge, P. E. Ciesielski, and W. H. Schubert, 1999: Trimodal characteristics of tropical convection. J. Climate, 12, 2397-2418, doi:10.1175/ 1520-0442(1999)012<2397:TCOTC>2.0.CO;2.

Khairoutdinov, M. F., and Y. L. Kogan, 1999: A large eddy simulation model with explicit microphysics: Validation against aircraft observations of a stratocumulus-topped boundary layer. J. Atmos. Sci., 56, 2115-2131, doi:10.1175 1520-0469(1999)056<2115:ALESMW > 2.0.CO;2.

$\longrightarrow$, and - 2000: A new cloud physics parameterization for large-eddy simulation models of marine stratocumulus. Mon Wea. Rev., 128, 229-243, doi:10.1175/1520-0493(2000)128<0229: ANCPPI $>2.0 . \mathrm{CO} ; 2$.

— , and D. A. Randall, 2002: Similarity of deep continental cumulus convection as revealed by a three-dimensional cloudresolving model. J. Atmos. Sci., 59, 2550-2566, doi:10.1175/ 1520-0469(2002)059<2550:SODCCC $>2.0 . C O ; 2$.

, and -2003 : Cloud resolving modeling of the ARM summer 1997 IOP: Model formulation, results, uncertainties, and sensitivities. J. Atmos. Sci., 60, 607-625, doi:10.1175/ 1520-0469(2003)060<0607:CRMOTA > 2.0.CO;2.
, S. K. Krueger, C.-H. Moeng, P. A. Bogenschultz, and D. A. Randall, 2009: Large-eddy simulation of marine deep tropical convection. J. Adv. Model. Earth Syst., 1 (15), doi:10.3894/ JAMES.2009.1.15.

Kogan, Y. L., 2013: A cumulus cloud microphysics parameterization for cloud-resolving models. J. Atmos. Sci., 70, 1423-1436, doi:10.1175/JAS-D-12-0183.1.

, and D. B. Mechem, 2014: A PDF-based microphysics parameterization for shallow cumulus cloud. J. Atmos. Sci., 71, 1070-1089, doi:10.1175/JAS-D-13-0193.1.

- M. P. Khairoutdinov, D. K. Lilly, Z. N. Kogan, and Q. Liu, 1995: Modeling of stratocumulus cloud layers in a large eddy simulation model with explicit microphysics. J. Atmos. Sci., 52, 2923-2940, doi:10.1175/1520-0469(1995)052<2923: MOSCLI>2.0.CO;2.

- D. B. Mechem, and K. Choi, 2012: Effects of sea-salt aerosols on precipitation in simulations of shallow cumulus. J. Atmos. Sci., 69, 463-483, doi:10.1175/JAS-D-11-031.1.

Larson, V. E., and J.-C. Golaz, 2005: Using probability density functions to derive consistent closure relationships among higher-order moments. Mon. Wea. Rev., 133, 1023-1042, doi:10.1175/MWR2902.1.

, R. Wood, P. R. Field, J.-C. Golaz, T. H. Vonder Harr, and W. R. Cotton, 2001: Systematic biases in the microphysics and thermodynamics of numerical models that ignore subgridscale variability. J. Atmos. Sci., 58, 1117-1128, doi:10.1175/ 1520-0469(2001)058<1117:SBITMA $>2.0 . C O ; 2$.

, J.-C. Golaz, and W. R. Cotton, 2002: Small-scale and mesoscale variability in cloudy boundary layers: Joint probability density functions. J. Atmos. Sci., 59, 3519-3539, doi:10.1175/ 1520-0469(2002)059<3519:SSAMVI >2.0.CO;2.

- D. P. Schanen, M. Wang, M. Ovchinnikov, and S. Ghan, 2012: PDF parameterization of boundary layer clouds in models with horizontal grid spacings from 2 to $16 \mathrm{~km}$. Mon. Wea. Rev., 140, 285-306, doi:10.1175/MWR-D-10-05059.1.

Lebsock, M., H. Morrison, and A. Gettelman, 2013: Microphysical implications of cloud-precipitation covariance derived from satellite remote sensing. J. Geophys. Res. Atmos., 118, 65216533, doi:10.1002/jgrd.50347.

Li, C., X. Jia, J. Ling, W. Zhou, and C. Zhang, 2009: Sensitivity of MJO simulations to diabatic heating profiles. Climate Dyn., 32, 167-187, doi:10.1007/s00382-008-0455-x.

Liu, C., and E. J. Zipser, 2009: "Warm rain" in the tropics: Seasonal and regional distributions based on $9 \mathrm{yr}$ of TRMM data. J. Climate, 22, 767-779, doi:10.1175/2008JCLI2641.1.

Luo, Z., G. Y. Liu, G. L. Stephens, and R. H. Johnson, 2009: Terminal versus transient cumulus congestus: A CloudSat perspective. Geophys. Res. Lett., 36, L05808, doi:10.1029/ 2008 GL036927.

Madden, R., and P. Julian, 1971: Detection of a 40-50 day oscillation in the zonal wind in the tropical Pacific. J. Atmos. Sci., 28, 702-708, doi:10.1175/1520-0469(1971)028<0702: DOADOI $>2.0 . \mathrm{CO} ; 2$.

— , and —- 1972: Description of global-scale circulation cells in the tropics with a 40-50-day period. J. Atmos. Sci., 29, 1109-1123, doi:10.1175/1520-0469(1972)029<1109: DOGSCC $>2.0 . \mathrm{CO} ; 2$.

Malkus, J. S., 1962: Large-scale interactions. Physical Oceanography, M. N. Hill, Ed., The Sea-Ideas and Observations on Progress in the Study of the Seas, Vol. 1, John Wiley and Sons, 88-294.

— Tropical Pacific Ocean. University of California Press, 229 pp. 
Mechem, D. B., and A. J. Oberthaler, 2013: Numerical simulation of tropical cumulus congestus during TOGA COARE. J. $A d v$. Model. Earth Syst., 5, 623-637, doi:10.1002/jame.20043.

- - P. C. Robinson, and Y. L. Kogan, 2006: Processing of cloud condensation nuclei by collision-coalescence in a mesoscale model. J. Geophys. Res., 111, D18204, doi:10.1029/2006JD007183.

Pincus, R., and S. A. Klein, 2000: Unresolved spatial variability and microphysical process rates in large-scale models. J. Geophys. Res., 105, 27 059-27 065, doi:10.1029/2000JD900504.

Rauber, R., H. T. Ochs III, L. Di Girolamo, S. Göke, and E. Snodgrass, 2007: Rain in Shallow Cumulus over the Ocean-The RICO campaign. Bull. Amer. Meteor. Soc., 88, 1912-1928, doi:10.1175/BAMS-88-12-1912.

Smolarkiewicz, P. K., and W. W. Grabowski, 1990: The multidimensional positive definite advection transport algorithm: Non-oscillatory option. J. Comput. Phys., 86, 355-375, doi:10.1016/0021-9991(90)90105-A.

Stephens, G. L., and N. B. Wood, 2007: Properties of tropical convection observed by millimeter-wave radar systems. Mon. Wea. Rev., 135, 821-842, doi:10.1175/MWR3321.1.
vanZanten, M. C., and Coauthors, 2011: Controls on precipitation and cloudiness in simulations of trade-wind cumulus as observed during RICO. J. Adv. Model. Earth Syst., 3, M06001, doi:10.1029/2011MS000056.

Waite, M. L., and B. Khouider, 2010: The deepening of tropical convection by congestus preconditioning. J. Atmos. Sci., 67, 2601-2615, doi:10.1175/2010JAS3357.1.

Wood, R., 2006: Rate of loss of cloud droplets by coalescence in warm clouds. J. Geophys. Res., 111, D21205, doi:10.1029/ 2006JD007553.

— P. R. Field, and W. R. Cotton, 2002: Autoconversion rate bias in stratiform boundary layer cloud parameterizations. Atmos. Res., 65, 109-128, doi:10.1016/ S0169-8095(02)00071-6.

Zhang, M. H., and J. L. Lin, 1997: Constrained variational analysis of sounding data based on column-integrated budgets of mass, heat, moisture, and momentum: Approach and application to ARM measurements. J. Atmos. Sci., 54, 1503-1524, doi:10.1175/1520-0469(1997)054<1503: CVAOSD $>2.0 . \mathrm{CO} ; 2$. 